\title{
Segmental arterial mediolysis: A clinical-pathologic review, its role in fibromuscular dysplasia and description and differential diagnosis of the masquerader-muscular artery cystic necrosis
}

\author{
Richard E. Slavin \\ Cascade Pathology Group, Department of Pathology, Emanuel Hospital and Health Center, Portland, USA \\ Email: rslavin@lhs.org, reslavin@comcast.net
}

Received 15 November 2012; revised 17 December 2012; accepted 28 December 2012

\begin{abstract}
Segmental arterial mediolysis (SAM) is an uncommon non-inflammatory iatrogenic arteriopathy caused by alpha-1 adrenergic agonists or Beta-2 agonists able to release norepinephrine from the peripheral nervous system. Causative agents include adrenergic agonists used to control blood pressure, B-2 tocolytic agonists, and ractopamine used as a repartitioning agent in animal husbandry. The liberated norepinephrine both injures and stimulates a robust reparative response in the muscular arteries in the abdomen, brain base, and coronary arteries. This response may be augmented by endothelin- 1 formed in the arterial adventitia. Three types of arterial lesions develop in the injurious stage: 1) apoptotic induced mediolysis, 2) separation of the outer media from the adventitia and 3) the formation of arterial gaps. The latter enlarge, particularly in elderly patients, to form gap-aneurysms complicated by dissections and dissecting aneurysms that when ruptured cause the calamitous hemorrhages that clinically announce SAM. The other types of injury remain clinically silent but with repair develop sequelae and can metamorphose into fibromuscular dysplasia. The sequelae are mainly asymptomatic but may cause arterial stenosis and ischemic lesions. The definitive diagnosis of SAM requires histological conformation but misinterpretation of smooth muscle vacuolar change has caused diagnostic errors. Muscular artery cystic necrosis a newly named non-inflammatory muscular artery arteriopathy may be confused with SAM both clinically and pathologically. This arteriopathy represents the muscular artery equivalent of cystic media necrosis of the elastic arteries since it exhibits similar morphologic features and can occur concomitantly with this entity. Adrenergic agents to counter hemorrhagic shock in SAM are contraindicated since they may
\end{abstract}

intensify injury and create new lesions. The use of norepinehrine antagonists introduces a new, but as yet untested, treatment option for SAM.

Keywords: Segmental Arterial Mediolysis; Norepinephrine; Adrenergic Agonists; Ractopamine; Arterial Apoptosis; Aneurysms; Dissecting Hematomas; Arterial Fibromuscular Dysplasia; Muscular Artery Cystic Necrosis

\section{INTRODUCTION}

Segmental arterial mediolysis (SAM), a rarely reported and not well recognized vascular lesion, can cause catastrophic hemorrhages from ruptured aneurysms and/or dissecting hematomas. Kernohan and Woltman described the first cases in adults as focal nonseptic necrosis of the vertebral and cerebellar arteries in 1943 [1]. Gruenwald in 1949 [2], and De SA 30 years later [3] reported it in the coronary arteries of stillborns, newborns and infants. Slavin \& Gonzalez-Vitale [4] recognized it as a distinct entity occurring in the abdominal muscular arteries of elderly patients and named it Segmental mediolytic arteritis since it was initially suspected as representing an immune-mediated arteritis. However, its localized nature, inconstant inflammatory response and an absence of concomitantly occurring laboratory evidence of an immunologic assault indicated that this hypothesis was incorrect $[5,6]$ and it was renamed SAM, a descriptive term derived from its most striking morphologic features [7]. Slavin and coworkers in a series of articles disclosed the various types of the lesions in SAM [4,5,7-9] their suspected evolution to arterial fibromuscular dysplasia (FMD) [7-9], ultimate fate [9]) and a proposed pathogenesis for this disorder [10]. This article will present a comprehensive analysis of this disease through a review of its pathology, radiological findings, pathogenesis, clinical presentation, prognosis, relationship to FMD, and 
treatment considerations. This assessment will also focus on the description and differential diagnosis of a newly named non-inflammatory muscular artery arteriopathy, muscular artery cystic necrosis that may masquerade as SAM [8].

\section{PATHOLOGY}

\subsection{Arterial Alterations}

Cognizance of the pathology of SAM is key to understanding its presentation, course, prognosis, radiological features and relationship to FMD. SAM is a disorder of the muscular arteries located in the abdomen, heart and brain base. Arterial lesions are divisible into two stagesan injurious phase followed by a reparative phase. These are not necessarily separate events since reparative changes may develop paripassu with injurious lesions. The alterations are segmental without partiality for branch points and may involve part, multiple segments of, or the entire arterial wall circumference. Typifying the injurious stage are three distinctive lesions: 1) mediolysis, 2) the severing of the adventitia from the outer media, and 3) the formation of arterial gaps from which aneurysms and dissecting hematomas may develop.

Mediolysis, the cardinal lesion of SAM, commences in the outer media. It can be restricted to this zone or may extend inwardly to involve most or all of the inner media. There are 2 pathways causing this lesion. The most prevalent one is characterized by the transformation of the cytoplasm of the involved smooth muscle into incompletely bordered zones of expanded clear cell sap with a foamy appearance caused by included minute vacuoles and membranous debris (Figures 1-3). The second, relatively rare, is the disintegration of the medial muscle cells into small granular cellular nuclear and cytoplasmic debris [10]. Ultrastructural examination of the first type of degenerative change reveals varying sized distended cytoplasmic vacuoles that apparently burst and disrupt cell membranes to cause mediolysis [5]. The liberated cell sap, that fails to stain with fat, PAS and alcian-blue stains, outwardly expand the internal and external elastica (Figure 3). Zones of mediolysis typically display fibrin deposits, scattered red cells and an occasional leukocyte putatively derived from distended and damaged vasovasorum (Figures 1, 4 and 5). Mediolysis is a transitory event and its resultant cellular debris may rapidly disappear (Figure 4) or be concealed by extensive fibrin deposits (Figure 5).

The initial lesion of SAM forms at the adventitial medial junction presenting as linear dense bands of fibrin often associated with microhemorrhages into the adjacent adventitia and outer media. This may be followed by an overt severing of the outer media from the adventitia (Figure 2). The created space contains individual intact

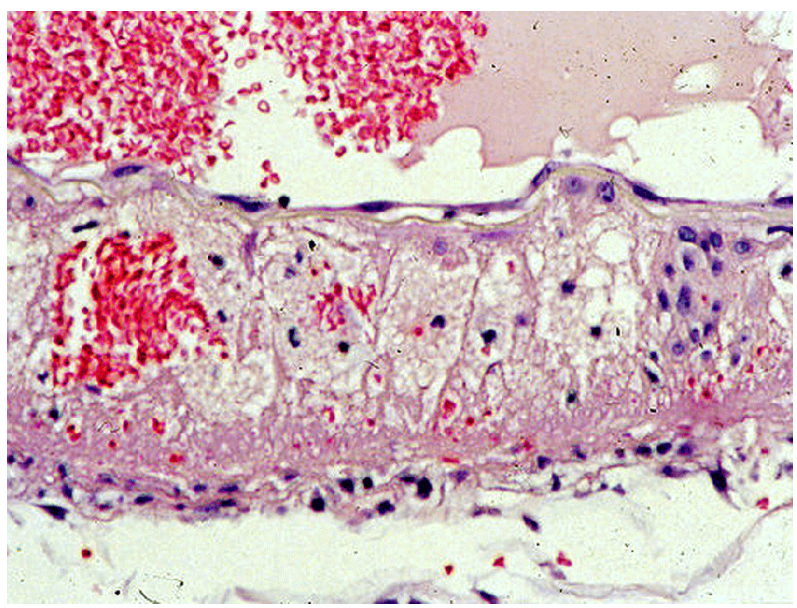

Figure 1. Injurious phase of segmental arterial mediolysis. Artery shows an area of almost transmural mediolysis exhibiting a foamy appearance produced by varying sized vacuoles and membranous remnants, an island of intact muscle cells, focally preserved subintimal muscle cells and intima, a focus of red cells, a few inflammatory cells and a linear band of fibrin at the adventitial medial junction. Hematoxylin and eosin $\times 200$. Adapted with permission from the International Journal of Surgical Pathology, 15(2) copyright 2007, Sage Publications, Inc.

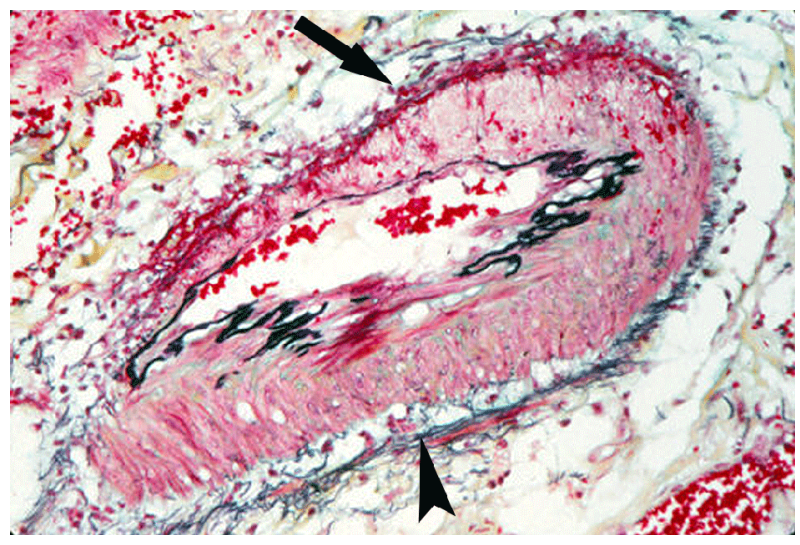

Figure 2. Injurious phase of segmental arterial mediolysis. Arrow shows an area of transmural mediolysis having a foamy appearance and bordered by an adventitial fibrin band and an intact internal elastica. Arrowhead segment of artery shows a tear separating of the outer media from the adventitia. The tear space contains a few intact muscle cells but does not have the foamy appearance of mediolysis. Movat stain $\times 100$. Adapted with permission from the International Journal of Surgical Pathology, 15(2) copyright 2007, Sage Publications, Inc.

separated muscle cells dispersed in expanded extracellular matrix material to which fibrin, red cells an edema fluid may be added. This tearing lesion generally accompanies mediolysis although foci of mediolysis may exist without evidence of separation and the reverse also may occur.

Arterial gaps of varying sizes are created by transmedial mediolysis and loss of the internal elastica and intima putatively caused by rending and loss of musculostromal 


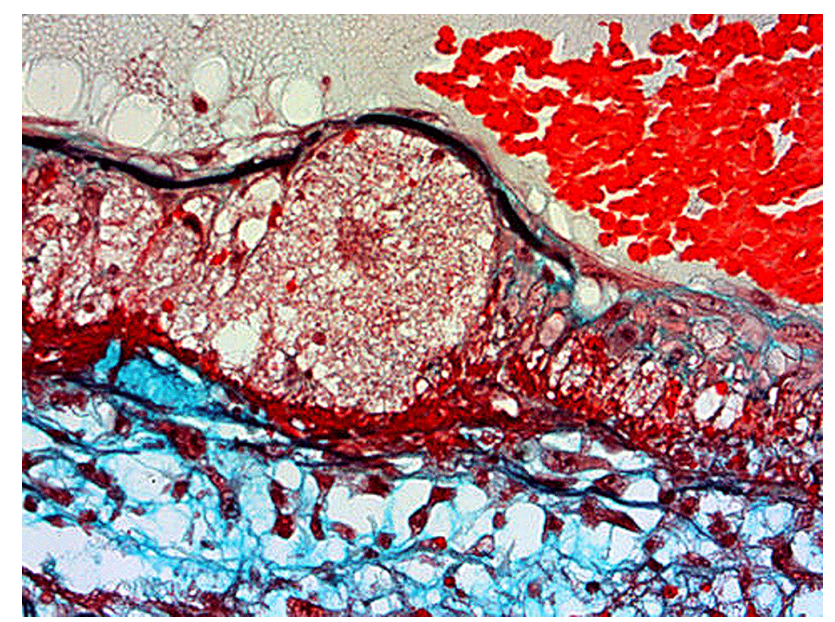

Figure 3. Injurious phase of segmental arterial mediolysis. Artery shows a focus of transmural mediolysis that has caused a bulging of the arterial wall bordered inwardly by an intact internal elastica and outwardly by a linear deposit of fibrin. The mediolytic zone has a foamy appearance and is alcian-blue negative. Movat stain $\times 200$. Adapted with permission from The American Journal of Surgical Pathology 13(7), copyright 1989, Lippincott, Williams and Wilkens.

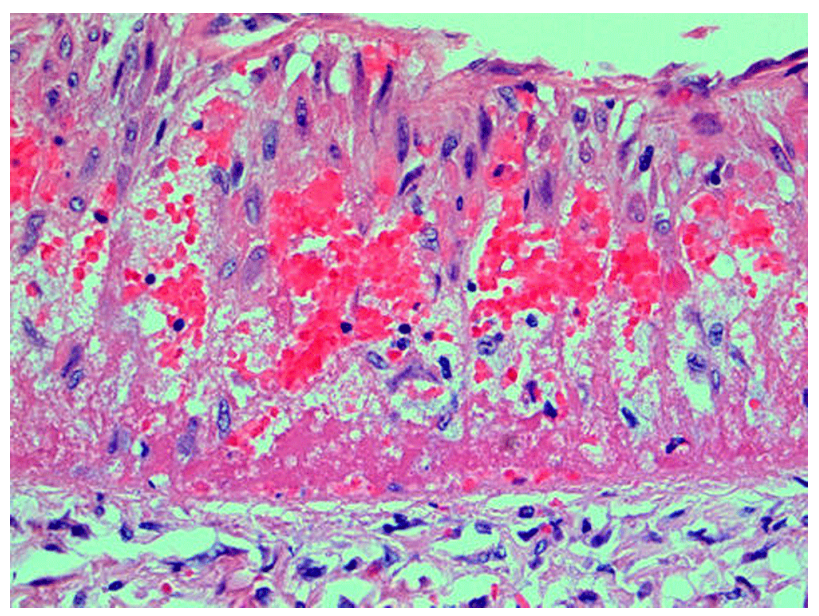

Figure 4. Injurious phase of segmental arterial mediolysis. Infant coronary artery showing mediolysis of the outer and mid media. Areas of mediolysis contain faint membranous and organelle debis, clusters of red cells, fibrin strands and a rare inflammatory cell. Scattered intact muscle cells are distributed in zones of mediolysis. A thick linear layer of fibrin borders the artery and extends into the outer media. Hematoxylin and eosin $\times 200$. Adapted with permission from Modern Pathology, 18(3), copright 1995, Nature publishing group.

connections (Figure 6). The gaps are outlined by the linear fibrin deposits initially formed at the adventitialmedial junction (Figure 7). The gaps often enlarge through the undermining and detachment of residual islands of adjacent intact arterial wall (Figure 7) [11]. Significant segments of the arterial wall can disappear in this process leading to the formation of saccular or elongated fusiform aneurysms (Figure 8). Thrombi may form

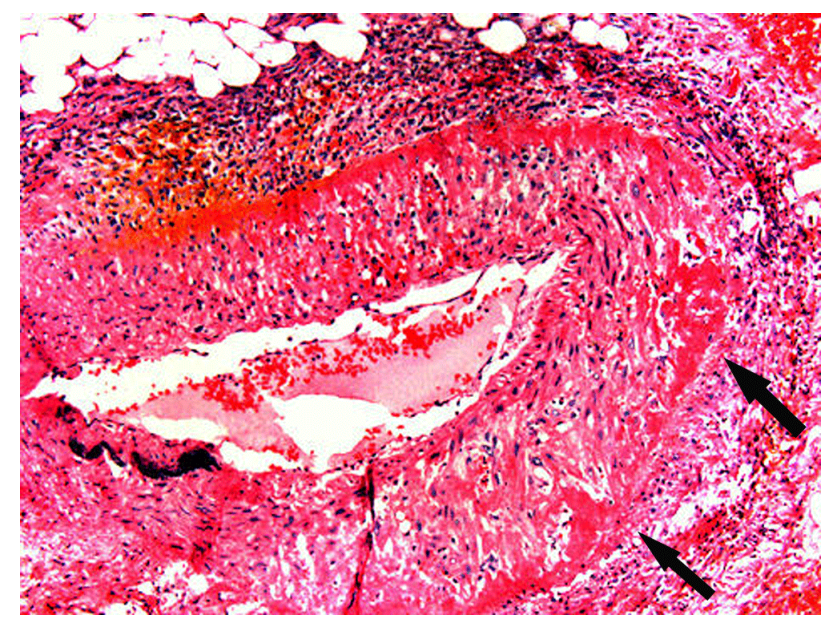

Figure 5. Injurious phase of segmental arterial mediolysis. Adventitial medial junction is circumscribed by a thick linear band of fibrin that extends into and fills areas of mediolysis (arrows) obscuring mediolytic induced cellular debis. Mononuclear cells, many fibroblasts are present in the upper periadventitial tissue. Hematoxylin and eosin $\times 50$.

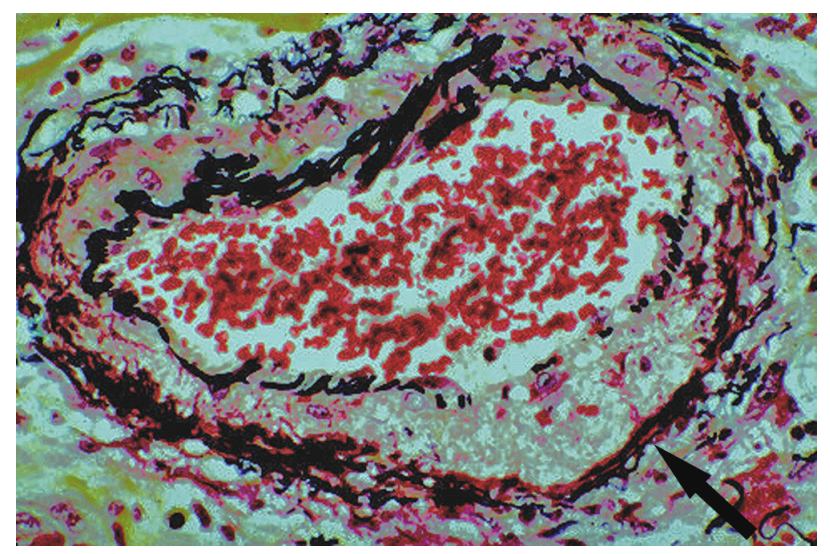

Figure 6. Injurious phase of segmental arterial mediolysis. Arrow points to an area of transmural mediolysis with overlying focal loss of the internal elastica-a prelude to gap formation. The mediolytic zone is foamy, alcian-blue negative and is bordered by a band of fibrin. Movat stain $\times 50$. Adapted with permission from The American Journal of Surgical Pathology 13(7), copyright 1989, Lippincott, Williams and Wilkens.

in some aneurysms (Figure 8) or in smaller arteries distal to those undergoing SAM. Complicating dissecting hematomas also arise. These are initiated at the gapintact media interface with the dissection proceeding between the outer media and the adventitia, utilizing the same pathway responsible for the detachment of medial islands (Figure 8). This creates a distinctive lesion-an artery with a double aneurysm (Figure 9).

In the reparative phase a robust, initially vascular-rich proliferation of granulation tissue fills and expands arterial wall defects, extends over adjacent intact intima to form plaques and fills the space created by the separation of the outer media from the adventitia (Figures $\mathbf{1 0}$ and 


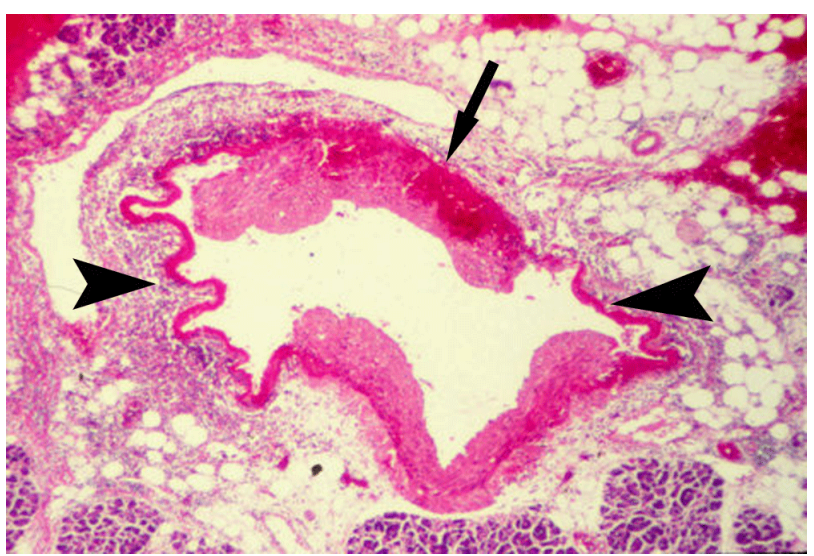

Figure 7. Injurious phase of segmental arterial mediolysis. Artery shows 2 gaps (arrow heads) bordered by linear bands of fibrin. Upper medial island (arrow) is being detached from the adventitia by blood tracking between the outer media and the adventitia. Its loss will significantly enlarge the arterial gap. Hematoxylin and eosin $\times 100$. Adapted with permission from the International Journal of Surgical Pathology, 15(2) copyright 2007, Sage Publications, Inc.

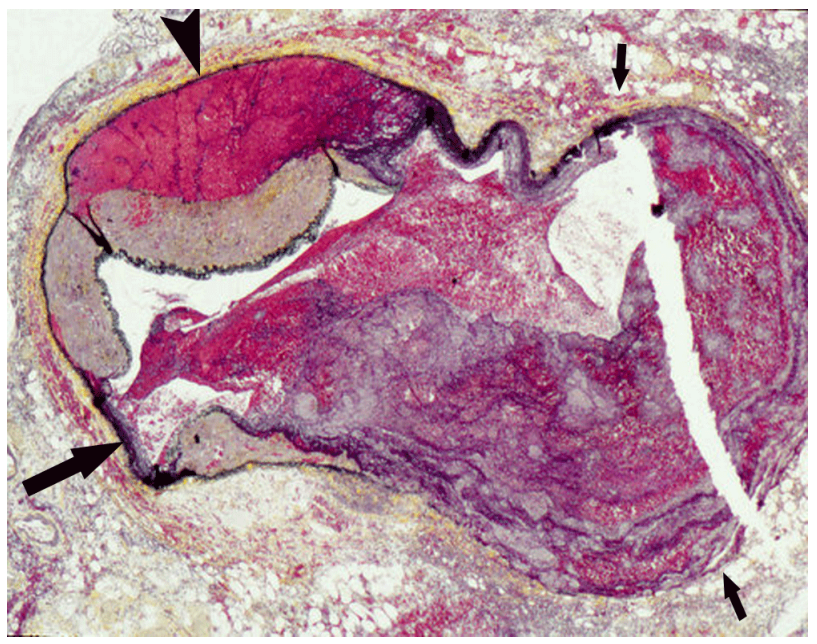

Figure 8. Injurious phase of segmental arterial mediolysis. Large gap aneurysm delineated by small arrows contains a recent thrombus. Large black arrow points to a small gap. Arrowhead points to a dissecting hematoma. Movat stain $\times 50$.

11). The capillaries in the early granulation tissue can dilate and assume grotesque shapes (Figure 10). These alterations may be accompanied by fibroblastic (Figure 10) and smooth muscle nodular proliferations in the periadventitial tissues. Bleeding from torn vessels in the granulation tissue causes medial hemorrhages and initiates the formation of hematomas and dissections in the separation plane between the outer media and adventitia (Figures 12 and 13). The organization of the granulation tissue repairs medial defects (Figure 14) obliterates small gaps and fortifies arterial aneurysms (Figure 15). It also may generate precursor lesions to FMD the type dependent upon its principal location in the artery. The

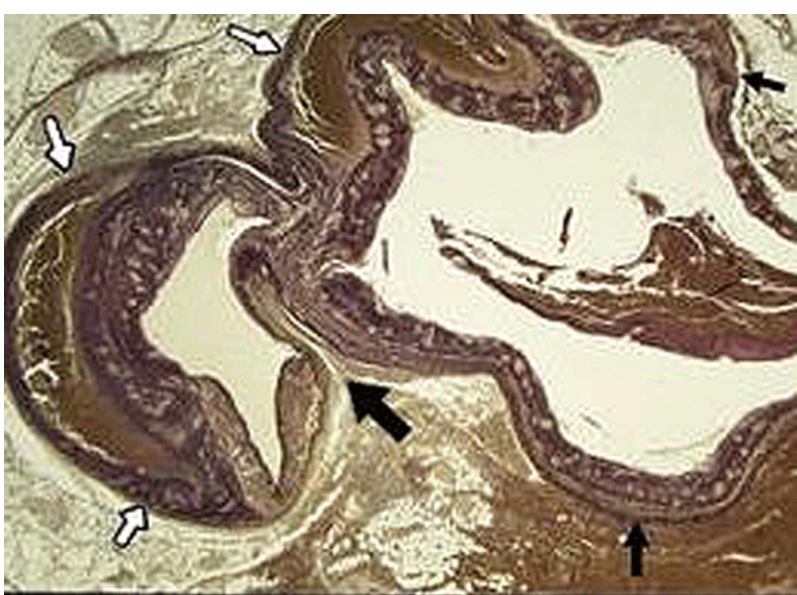

Figure 9. Injurious phase of segmental arterial mediolysis. Small black arrows demarcate a huge gap-aneurysm and white arrows point to a dissecting aneurysm-the double aneurysm of SAM. Large black arrow points to a medial gap interface showing an early separation of the outer media from the adventitia. Movat stain $\times 15$. Adapted with permission Cardiovascular Pathology, 18(6), Copyright 2009, Elsevier Inc.

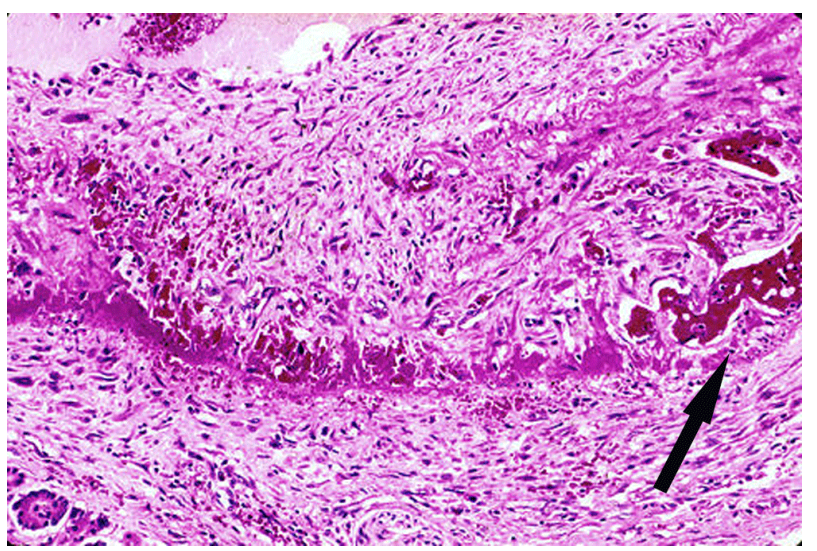

Figure 10. Reparative phase of segmental arterial mediolysis. Young granulation tissue, rich in capillaries, reoccupies an area of transmural mediolysis and extends into adventitia and over the adjacent intima to form a plaque. Arrow points to dilated irregular shaped capillary. Hematoxylin and eosin $\times 200$. Adapted with permission from the International Journal of Surgical Pathology, 15(2) copyright 2007, Sage Publications, Inc.

organization of granulation tissue in the adventitia creates adventitial fibroplasia (Figure 16), in the tear between the outer media and adventitia fashions perimedial dysplasia (Figure 17), and if developing in significant zones of medial muscle loss generates medial dysplasia (Figures 11 and 18).

\subsection{Venous Alterations}

A venous angiopathy occurs in the abdominal veins located immediately adjacent to large arteries showing changes of SAM (Figure 19) [8,12]. It features irregularly distributed vacuolar change of the cytoplasm of the 


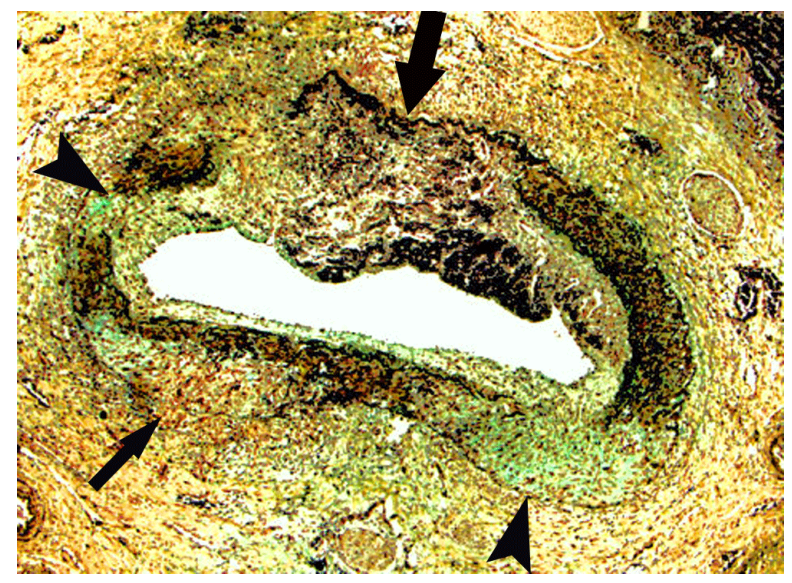

Figure 11. Reparative phase of segmental arteral mediolysis. Granulation tissue repairing a focus of partial mediolysis (small arrow), two foci of total mediolysis (arrowheads) and filling a large gap (large arrow). The granulation tissue expands to cause bowing of the external elastica and extends over the intima to form an intimal plaque. Attached to the granulation tissue filling the gap is a recent thrombus. Movat stain $\times 50$. Adapted with permission from Modern Pathology, 18(3), copyright 1995, Nature Publishing group.

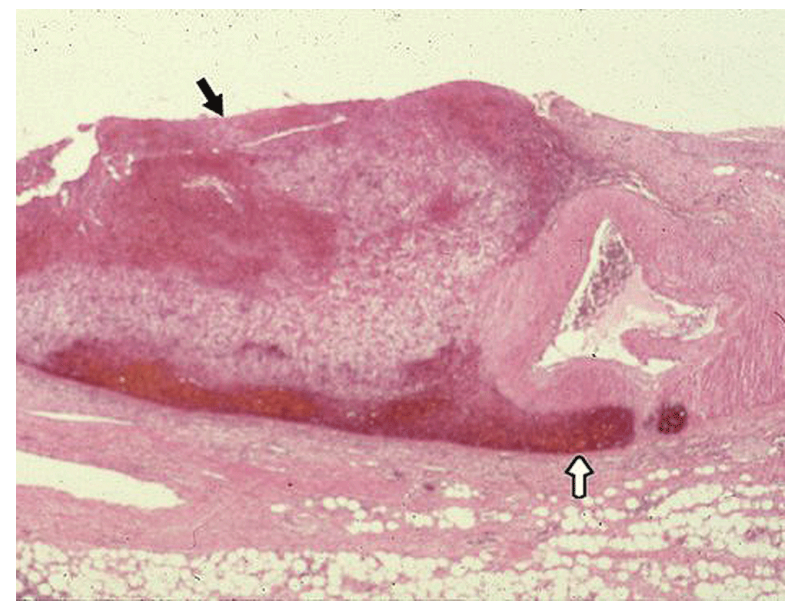

Figure 12. Reparative phase of segmental arterial mediolysis. Black arrow points to a florid proliferation of young granulation tissue in the space created by the tearing separation of the outer media from the adventitia. Capillary bleeding has initiated a dissecting hematoma (white arrow) tracking between the outer media and the adventitia. Hematoxylin and eoain $\times 15$. Adapted with permission from the International Journal of Surgical Pathology, 15(2) copyright 2007, Sage Publications, Inc.

medial smooth muscle with in some veins concomitant patchy loss of the internal elastica (Figure 20). The lesions are segmental and partially or totally circumferential. The vacuoles initially small, expand to occupy all of the cytoplasm of the muscle cells. Its contents are clear and PAS, alcian-blue and fat negative. Mediolysis is caused by the dissolution of the cell membranes of these cells (Figure 21). Not all muscle cells exhibit this degenerative sequence. Some show cytoplasmic hy-

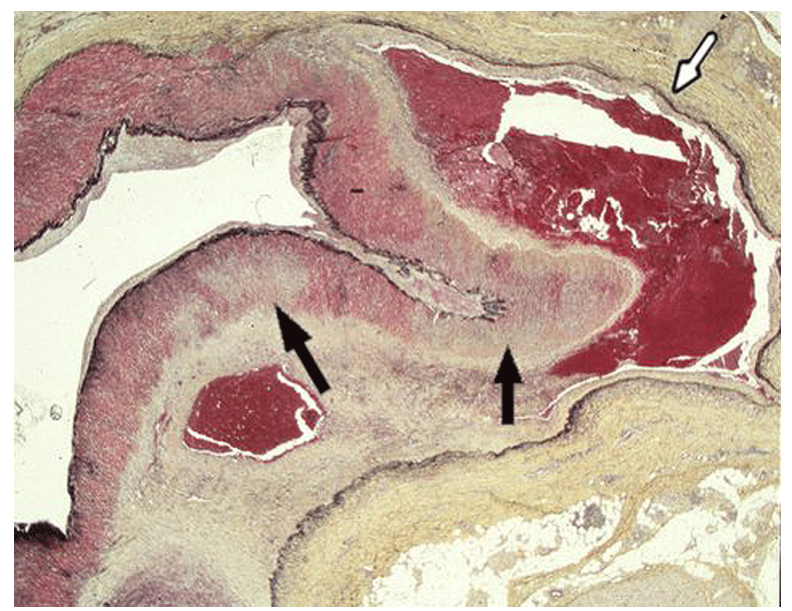

Figure 13. Reparative phase of segmental arterial mediolysis. Dissecting hematoma (white arrow) and hematoma developed in the mature granulation tissue filling and expanding the separated space between the outer media and adventitia. Black arrows point to areas of mediolysis replaced by mature granulation tissue. Movat stain $\times 15$. Adapted with permission from the International Journal of Surgical Pathology, 15(2) copyright 2007, Sage Publications, Inc.

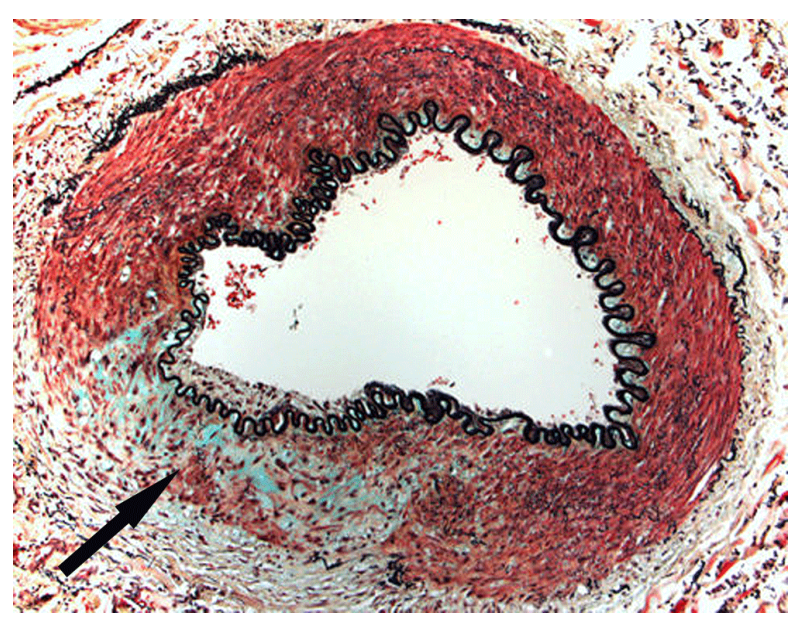

Figure 14. Reparative phase of segmental arterial mediolysis. Arrow points to an irregular focus of granulation tissue replacing an area of mediolysis.The internal elastica is intact and a thin intimal plaque is evident overlying the repaired focus of mediolysis. Such lesions are asymptomatic and on angiograms will appear "resolved”. Movat Stain $\times 100$. Adapted with permission from Cardiovascular Pathology, 18(6), copyright 2009, Elsevier Inc.

pereosinophilia, shrinkage and fragmentation associated with nuclear pyknosis and loss (Figure 21). The released vacuolar contents, commingled with membranous and cytoplasmic fragments and occasional polymorphonuclear cells and erythrocytes, causes venous edema that widely separates the surviving medial muscle and disrupts the intima with narrowing of the lumen (Figure 22). The medial muscle loss produces irregular outer medial wall contours (Figure 19), a "moth-eaten" media or, if 


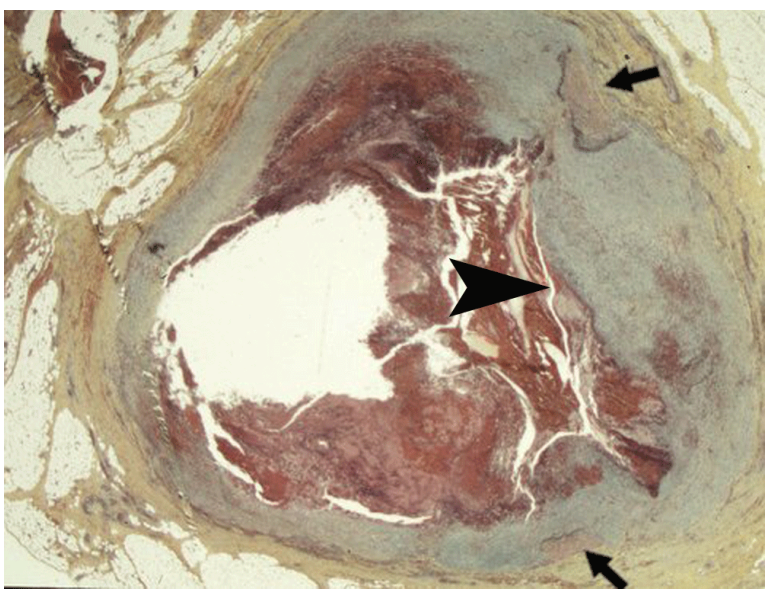

Figure 15. Reparative phase of segmental arterial mediolysis. Arrowhead points to a thick layer of granulation tissue filling a large gap and extending over the intimia of the surviving arterial wall islands (arrows) to encircle an adjacent large gap aneurysm. Lumen contains a recent thrombus. Movat stain $\times 25$. Adapted with permission from Cardiovascular Pathology, 18(6), copyright 2009, Elsevier Inc.

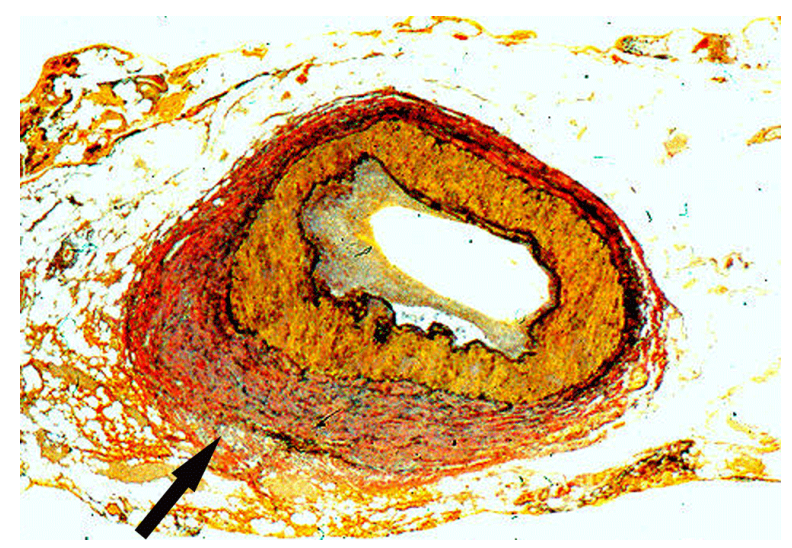

Figure 16. Reparative phase of segmental arterial mediolysis. Arrow points to an irregular area of adventitial fibrosis indistinguishable from periadventitial fibromuscular dysplasia. Verhoeff-van Gieson ×50.

transmedial with intimal loss, defects results in perivenous hemorrhages and/or formation of luminal thrombi. Areas of medial loss repaired by fibrosis may create a medial FMD-like venous lesion (Figure 23) [13].

Preliminary studies show endothelin-1 (ET-1) staining the membranous debris in areas of mediolysis and cell membranes of the intact medial venous muscle and venous intima (Figure 24). ET-1 is also detected in the adventitial capillaries of adjacent arteries (Figure 25) but unlike the veins this agent is not found in their arterial wall [8].

\section{PATHOLOGIC-RADIOLOGIC CORRELATIONS}

The six different angiographic images detected in SAM

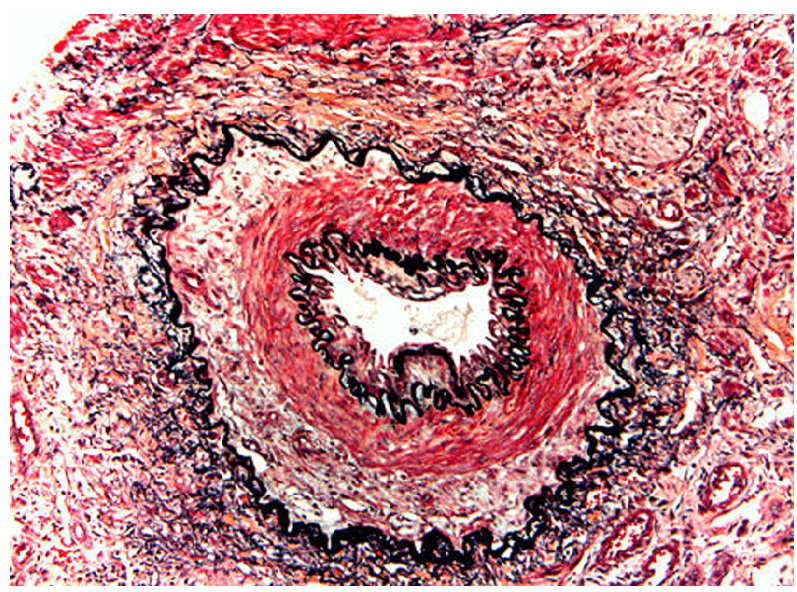

Figure 17. Reparative phase of segmental arterial mediolysis. Mature granulation tissue fills tear space between outer media and adventitia. Fibrosis of this tissue will lead to perimedial fibromuscular dysplasia. Movat stain $\times 100$. Adapted with permission from the International Journal of Surgical Pathology, 15(2) copyright 2007, Sage Publications, Inc.

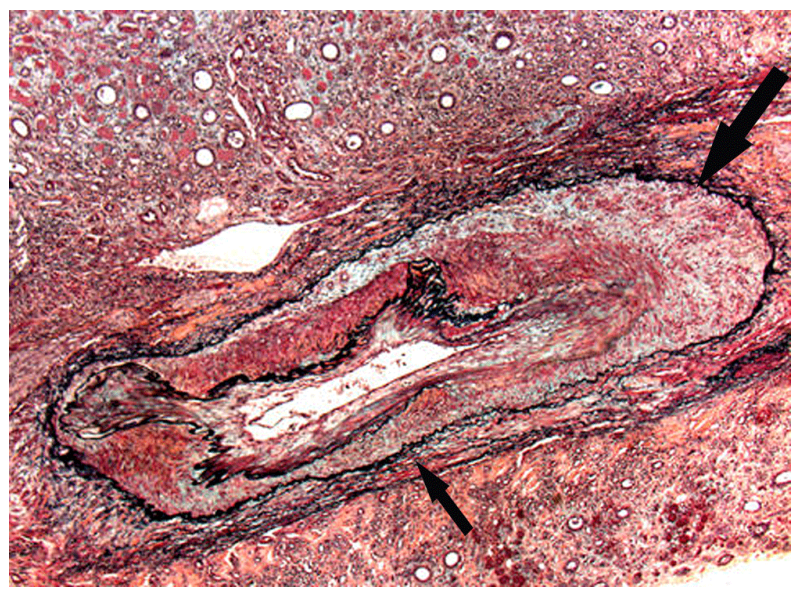

Figure 18. Reparative phase of segmental arterial mediolysis. Arrow points to mature granulation tissue filling and expanding the space between the outer media and external elastica and replacing areas of medial muscle loss most pronounced in the lower portion of the artery (small arrow). The internal elastica is intact and bedecked by a circumferential thin plaque of granulation tissue. Fibrous maturation of the granulation tissue will fashion medial fibroplasias. Movat stain $\times 25$. Adapted with permission from Cardiovascular Pathology, 18 (6), copyright 2009. Elsevier Inc.

are arterial dilatations, singular and multiple aneurysms, dissecting hematomas, arterial stenosis and arterial occlusions [9]. These mirror the kaleidoscope of lesions created in the injurious and reparative stages of SAM. Arterial dilatations are caused by mediolysis that depletes the media of supporting smooth muscle. With the additional loss of the internal elastica and intima, gaps form and with their enlargement aneurysms develop. Several adjacent gap forming aneurysms, created by the segmental distribution of SAM, generates the string-of-beads 


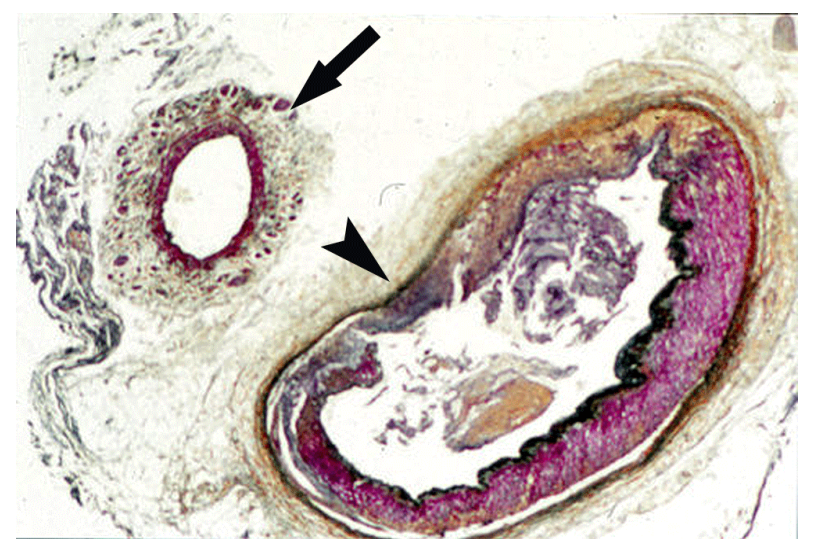

Figure 19. Venous angiopathy accompanying segmental arterial mediolysis. Outer media of venous angiopathy (arrow) exhibits a ragged contour due to the irregular mediolytic loss of mid and outer muscle bundles. Despite muscle loss, the vein is thickened by membranous debris and edema fluid filling areas of muscle loss. The adjacent artery shows a gap (arrow head) filled with granulation tissue that extends upwardly to repair a zone of transmural mediolysis and downwardly to fill an adventitial medial tear space. Movat stain $\times 25$.

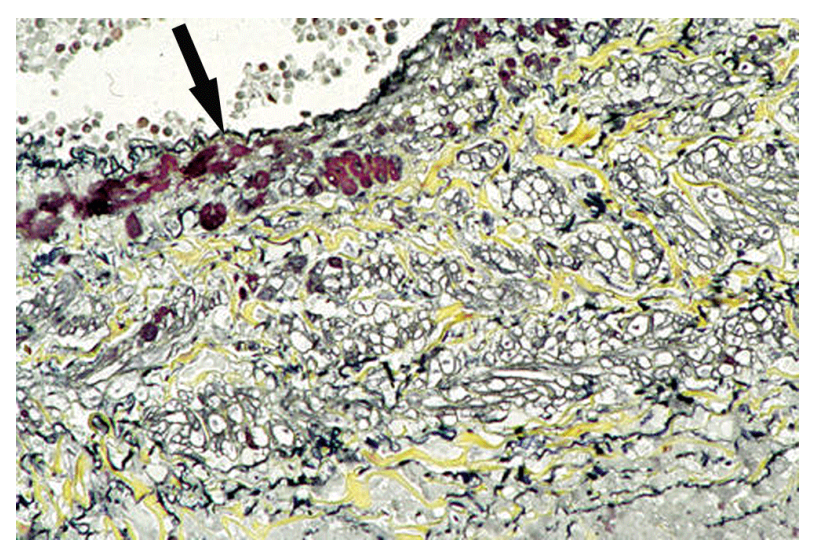

Figure 20. Venous angiopathy accompanying segmental arterial mediolysis. Smooth muscle of venous media show vacuolar degenerative change. Arrow points to scattered intact subintimal muscle cells. Movat stain $\times 200$.

appearance. These angiographic images occur in injurious stage SAM. The reparative phase features persisting nonruptured aneurysms that may decrease in size and even disappear and arterial stenosis, a sequela detected weeks or months after the onset of SAM, produced by intimal plaques and/or hematomas or dissections in the arterial wall. Two types of dissections develop in SAM both without benefit of intimal tears and both forming between the outer media and adventitia. The one fashioned in the injurious phase is often a unique lesion composed of two types of aneurysms-the double aneurysm of SAM (Figure 8) while the other type occurring in the reparative phase, originates from bleeding in the granulation tissue filling the space fashioned by the adventitial medial separation. This latter can occur

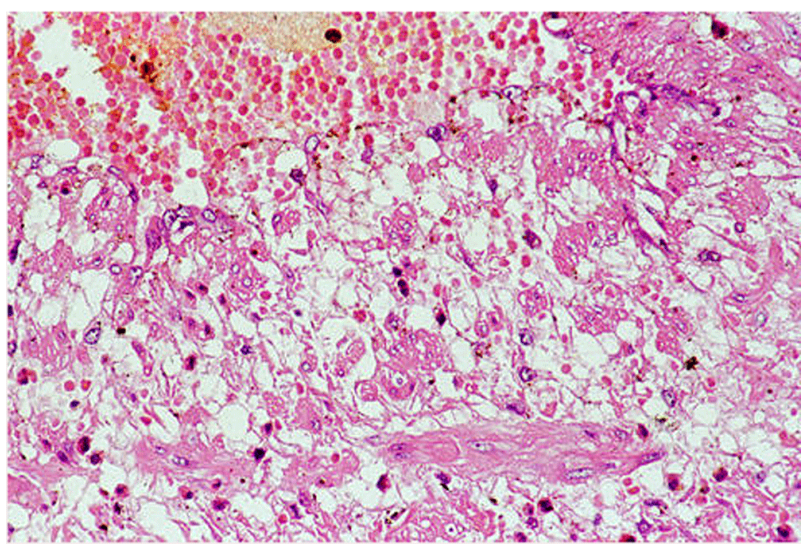

Figure 21. Venous angiopathy accompanying segmental arterial mediolysis. Mediolysis is created by the rupture of the large vacuoles in the cytoplasm of the smooth muscle cells. Scattered intact muscle cells show small vacuoles while a few are tiny with hypereosinophilic cytoplasm and pyknotic nuclei. Hematoxylin and eosin $\times 200$. Adapted with permission from International Journal of Surgical Pathology, 15(2) copyright 2007, Sage Publications, Inc.

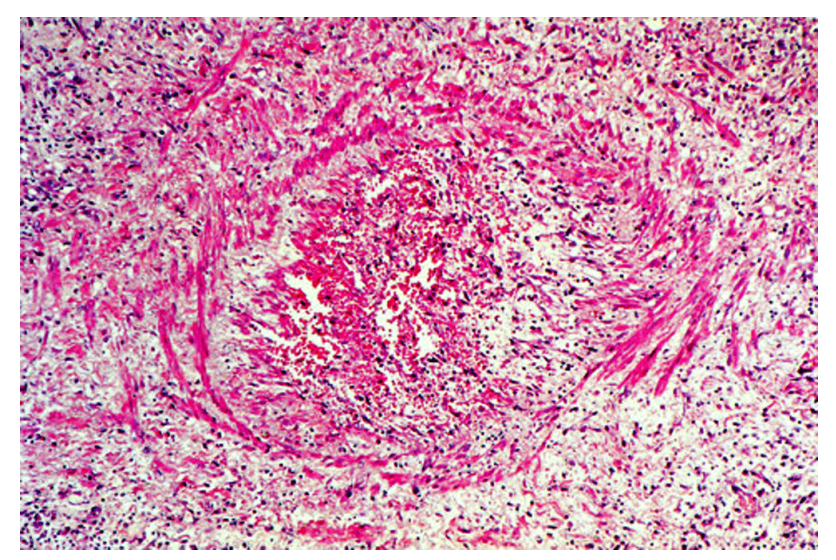

Figure 22. Venous angiopathy accompanying segmental arterial mediolysis. Watery fluid released by mediolysis, containing cellular debris, widely separates surviving medial muscle and expands and disrupts the venous intima. Lumen contains a recent thombus. Hematoxylin and eosin $\times 100$.

immediately following the injurious stage (Figure 12) but typically develops 1 to 1.5 months after the onset of SAM (Figure 13), sometimes more than one year latter [14]. Thrombi are the principal cause of arterial occlusions. They develop in large gap-aneurysms, in arterial lumens narrowed by reparative changes and in arterial branches distal to those exhibiting SAM where they can cause focal infarcts. Thrombi form in both the injurious and reparative phases of SAM. They may either recanalize or persist to be circumvented by a collateral circulation.

\section{PATHOGENESIS}

SAM is not an infectious, immunological, degenerative or congenital disorder. Slavin and coworkers initially 


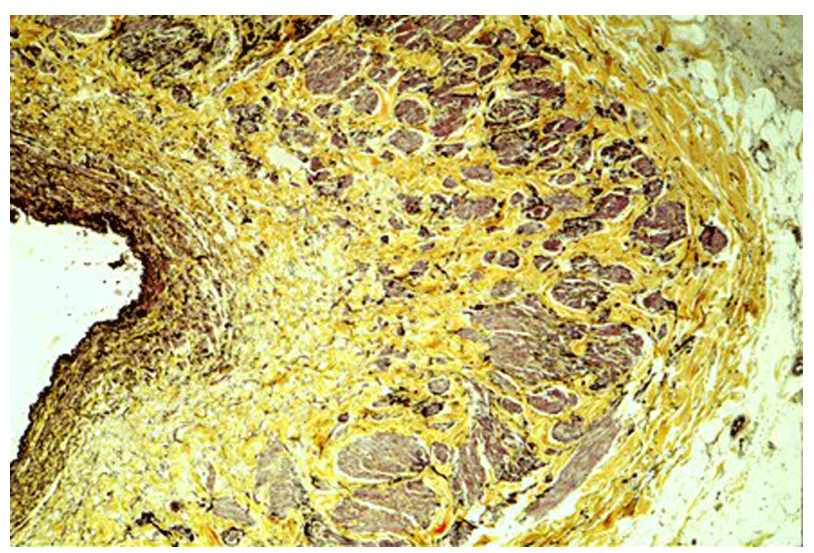

Figure 23. Venous angiopathy accompanying segmental arterial mediolysis. Medial muscle loss with replacement fibrosis principally occurring in the inner and mid media with general preservation of the outer media - a distribution pattern different from that observed in SAM. The fibrosis creates a venous medial fibrodysplastic-like lesion. Movat stain $\times 100$. Adapted with permission from the International Journal of Surgical Pathology, 15(2) copyright 2007, Sage Publications, Inc.

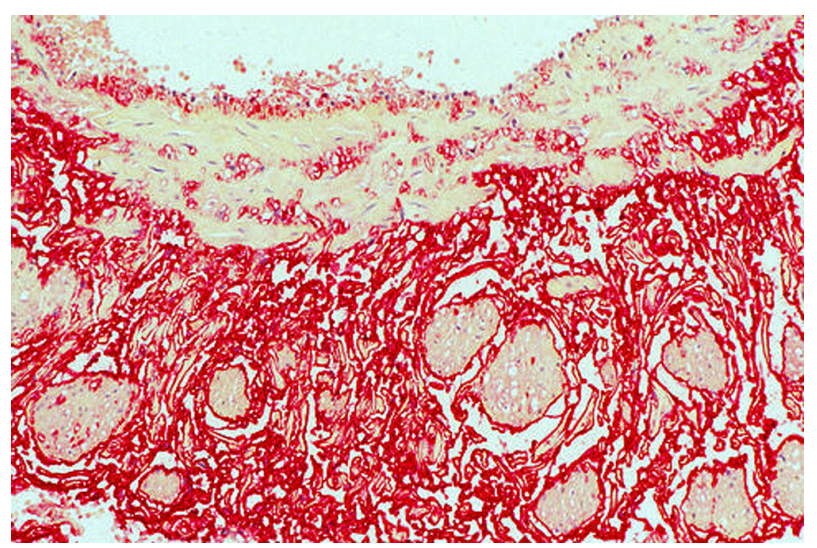

Figure 24. Venous angiopathy accompanying segmental arterial mediolysis. ET-1 (red stain) stains the intima, medial muscle cell membranes, membranous material in the out media and capillaries. ET-1 immunostain $\times 100$. Adapted with permission from the International Journal of Surgical Pathology, 15(2) copyright 2007, Sage Publications Inc.

proposed that it represented a vasospastic malady caused by norepinephrine because of its morphological characteristics and the initial site of injury at the adventitialmedial junction [5,7-9]. This hypothesis was validated by the discovery of SAM in the abdominal and coronary arteries of greyhound dogs following a single dose of ractopamine a Beta-2 agonist used as a repartitioning agent in animal husbandry and as an illicit agent to enhance athletic performance in racing animals [10]. The repartitioning properties insure a muscular carcass in leaner animals by dramatically building muscle by increasing protein synthesis, inhibiting fat production and inducing lipolysis [15]. This muscle creating trait may explain the formation of nodules of smooth muscle

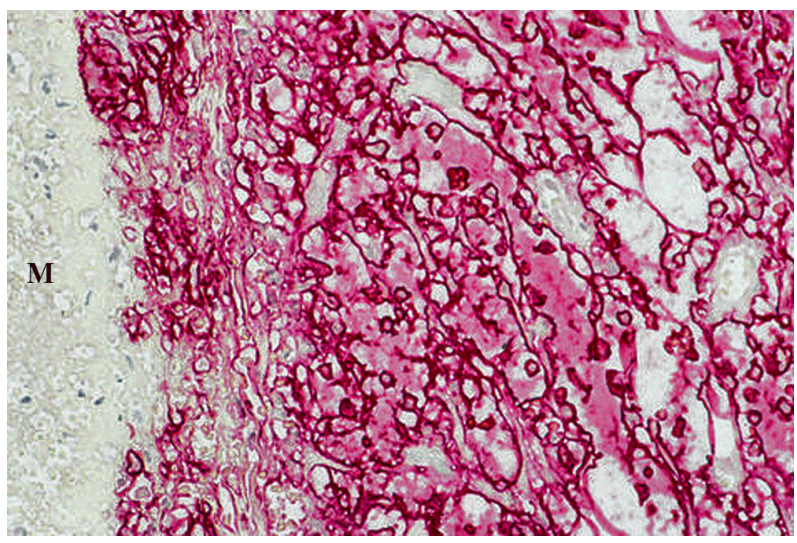

Figure 25. Venous angiopathy accompanying segmental arterial mediolysis. ET-1 in the arterial adventitia outlines capillaries and the cytoplasm of the endothelial cells projecting into the capillary lumens. M indicates arterial media. ET-1 immunostain $\times 200$. Adapted with permission from the International Journal of Surgical Pathology, 15(2) copyright 2007, Sage Publications, Inc.

that are occasionally discovered in and around the adventitia of SAM [10]. Ractopamine also enhances athletic performance by both its B-adrenergic agonist properties and anabolic activities. It is believed that ractopamine like clenbuterol, another B-2 agonist with very similar pharmacological properties, has the ability to neuromodulate the peripheral sympathetic system to release norepinephrine [16]. The liberated norepinephrine produced in the cardiac sympathetic ganglia stimulates the cardiomyocyte B-1 adrenergic receptors to induce myocardial apoptosis, a prominent findings in these canines [17] and the released norepinephrine manufactured in the varicosities of the efferent nerves serving the splanchnic arteries stimulates the alpha-I receptors on the cell membrane of the medial smooth muscle to either cause transient intense vasoconstriction followed by apoptosis and/or directly initiates apoptosis through the shearing separation of the adventitia from the media. This latter mechanism is believed responsible for the earliest lesion of SAM - the fibrin deposits and bleeding at the adventitial medial junction. Putatively the initial high levels of ractopamine enabled the activation of the alpha receptors by norepinephrine to override the stimulation of the B2 receptors by this agonist. The certain recognition of norepinephrine as the culprit responsible for SAM clarifies the genesis of arterial mediolysis. The vacuolar alterations in the arterial and venous medial muscle represents a stage of mitochondrial-mediated apoptosis (intrinsic pathway) caused by the opening of the mitochondrial permeability transitional pore allowing water to enter the mitochondria causing the cytoplasmic vacuolar change [18]. The rupture of the distended mitochondria floods the cytoplasm with water that disrupts the cell membrane resulting in mediolysis and the transitory outward bulging of the internal and/or external elastica in 
the affected arteries and the "edema” separating muscle bundles in adjacent veins. The second pathway to mediolysis-granular cellular fragmentation and loss represents the more classic endpoint in the intrinsic apoptotic pathway. Both types of apoptotic changes may occur in SAM and its accompanying venous angiopathy.

The realization that ractopamine can cause SAM is the Rosetta stone in our understanding of its pathogenesis. Perplexing was the occurrence of SAM in widely disparate patient populations - the elderly with perturbations in vasoregulation $[4,7,8]$, in new-born and infants, many prematurely delivered [2,3,5], in adults following operative procedures $[1,4,5,7]$, and most puzzling of all, cases seen in consultation developing unexpectedly in apparently healthy individuals. This conundrum can be resolved by considering SAM as a disorder principally or totally caused by iatrogenic or accidental exposure to alpha-1 adrenergic receptor agonists or Beta-2 agonists with peripheral sympathomimetic properties [10]. The major causative agents include adrenergic agonists used to control blood pressure, B-2 tocolytic agonists and the ingestion of meat and poultry products contaminated with ractopamine and clenbuterol and diet, athletic enhancing products, and illicit drugs [19] containing either one of these latter 2 agents. Representative examples supporting this hypothesis were discovered in prior publications. SAM was encountered in the coronary arteries of a 28-week old fetus, one twin in a twin transfusion syndrome, autopsied one day after tocolysis, amnioncentesis, fetal death and cesarean section [8]. The drug administered for tocolysis is unknown but the findings of early injurious stage SAM in the large intramural coronary arteries strongly suggest that it was one of the Beta-2 tocolytic agonists-fenoterol, terbutaline or ritodrine-utilized to relax uterine muscle. These drugs exhibit Beta- 1 cardiac activity and can pass from the placenta to the fetus and cause myocardial necrosis [20]. The myocardium of this fetus showed necrosis. Seventy percent of infant cases with SAM reported by De Sa [3] occurred in stillborns or premature infants. Although tocolysis was not mentioned in their histories, we suspect, on the basis of the patient population, that many mothers of these infants were administered B-2 tocolytic agonists. Coronary artery SAM in this study was also accompanied by myocardial necrosis, a finding described by Yaeger and coworkers in the ractopamine fed greyhound [17]. Injurious stage SAM also was detected in the submucosal arteries of a resected segment of small bowel in a patient treated with dopamine for hypotension administered 1 day before surgery performed for adhesion induced intestinal obstruction (Figure 1) [8]. It was also uncovered in the large hepatic arteries of a 75 year old female in heart failure who died less than 1 day after receiving dopamine for a hypotensive event [21] and in the coronary arteries of an infant following 2 weeks of continuous low dose dopamine treatment for renal dysfunction (Figure 4) [7]. Dopamine, an immediate metabolic precursor to norepinephrine, in high dosage elevates blood pressure through activation of the alpha-1 receptors. The prolonged period of treatment with the low-dose dopamine in the infant permitted high dose serum levels to accumulated accounting for the late development of early SAM found in the autopsied child. Kernohan and Woltman reported lesions in the vertebral and posterior cerebellar arteries, consistent with injurious stage SAM, in 4 patients developing 1 to 4 days postsurgery [1]. Two of these patients had spinal anesthesia. We suspect that drugs such as phenylephrine commonly administered to combat hypotension during surgery especially with spinal anesthesia may have caused SAM since this agent also exhibits a postsynaptic alpha-adrenergic effect.

The identification of ET-1 in adventitial tissues immediately adjacent to SAM and in contiguous abdominal veins suggests that this agent also plays a role in the genesis of SAM. This potent vasoconstrictor, by crosstalking with norepinephrine produced in the ganglia of the efferent sympathetic nerve endings in the arterial adventitia, can potentiate its pressor activity [22], thereby turning a physiologic stimulus into an injurious lesion. The failure of SAM to develop in the many persons who take or ingest iatrogenic adrenergic agents may be related to low dosage, a lack of sensitivity to these agents or to an absence of this amplification. ET-1 also promotes the formation of granulation tissue [23] thereby augmenting the robust reparative response that typifies SAM and develops almost lockstep with the injurious lesions. The venous angiopathy accompanying SAM shares many morphologic features of SAM suggesting that this angiopathy is also provoked by norepinephrine. However, the presence of ET-1 on the medial muscle membranes, membranous fragments and endothelium with its absence in these locations in the adjoining arteries showing SAM, the non-occurrence of the reactive components of SAM in the venous media, the variable location of the initial sites of mediolysis in the venous angiopathy in contradistinction to its initial strict occurrence in the outer media in SAM and finally its very sporadic appearance and predominant localization to large abdominal veins hints that the venous changes are induced by ET-1. It is morphologically similar to SAM since both lesions represent a vascular stereotypic response to pressor induced vasospasm.

\section{CLINICAL CONSIDERATIONS}

SAM is an acute, self-limiting non-systemic disorder generally localized to specific sites most commonly developing in adults in the abdominal muscular arteries and 
the arteries at the base of brain. Simultaneous development of SAM at both these sites has been reported [24,25] one case representing a possible recurrence of SAM an event explainable by the re-exposure to a causative adrenergic agonist. SAM's abdominal presentation is announced by abdominal apoplexy-a syndrome featureing abdominal pain and distention, rapidly falling hematocrit and hypovolemic shock caused by significant bleeding into the peritoneal cavity and/or retroperitoneum. These hemorrhages often carry a 50\% mortality rate [26], but with timely surgery the prognosis is good [27]. Accompanying laboratory evaluation implicating a vasculitis is negative. The injurious stage lesions causing this hemorrhage develop very rapidly, already being morphologically evident less than one day following the ingestion or administration of the etiologic agonist. Hemorrhage frequently stems from a single artery although simultaneous hemorrhage from more than one artery, often derived from different major abdominal arterial trunks can occur. The celiac, superior, and inferior mesenteric arteries and all their branches as well as the distal renal artery may be targeted but the most frequently involved arteries in one series were the splenic, hepatic and the distal renal arteries and their immediate branches [9] while in another series that excluded the hepatic and celiac axis, it was the middle colic, gastric and gastroepiploic arteries [27]. Frequently, some of the immediate tributaries of the involved arteries, or only these branches will exhibit SAM, for example the large pancreatic branches of the splenic artery, the large intrahepatic branches of the hepatic artery, the hilar, lobar and arcuate branches of the renal artery, and the serosal and submucosal branches of the intestinal arteries. Arterial involvement is usually limited to a small area in a participating abdominal organ a feature negating extensive organ resection and for the diagnostic consideration of SAM in organs exhibiting multiple peripheral aneurysms.

In about $1 / 3$ to $3 / 4$ of reported cases of abdominal SAM more than one artery is assaulted [9] but those not developing gap-aneurysms, the major cause of hemorrhage, are initially asymptomatic. The fate of the nonbleeding injured arteries is revealed by angiographic follow-ups performed weeks, months, and even years after the onset of SAM [9]. Damaged arteries may be completely remodeled by the reparative granulation tissue and appear "normal" in subsequent angiographic studies (Figure 14). Others will exhibit sequelae that include dissecting hematomas, arterial stenosis, late arterial occlusions and FMD that may create ischemic lesions. However, except for late dissections, sequelae, in the small number of reported cases, have been asymptomatic. Aneurysms may persistent for 3 years in one angiographic follow-up [28] but hemorrhage from persevering aneurysms has not been reported to date but large aneurysms potentially may rupture so that periodic surveillance is prudent. The finding of repaired arteries and asymptomatic sequelae indicates that SAM may be entirely subclinical and therefore more common than the few reported cases would indicate.

Occasionally abdominal SAM may not present with massive bleeding, but with the affects of organ injury caused by complicating arterial thrombosis. In this manner hematuria, caused by renal cortical infarcts can announce SAM (see case 8). As indicated above ischemic bowel lesions caused by sequelae induced arterial narrowing also may be a presenting symptom of SAM.

Coronary artery involvement principally develops in utero, in newborns, in infants and children.

Rare cases also have been reported in young adults [29]. It involves the extramural coronary arteries and their immediate large perforating branches. Gap-aneurysms are not a feature of coronary artery SAM but dissections can occur [29]. Symptoms may develop as a consequence of accompanying myocardial necrosis caused by norepinephrine induced apoptosis followed by fine reparative fibrosis or complicating coronary artery thrombi $[3,17]$.

Central nervous system (CNS) SAM presenting with subarachnoid hemorrhages occurs in adults and involves the arteries at the base of the brain. In the small number of cases reported these most often develop from gapaneurysms or dissections in the vertebral, posterior inferior cerebellar and internal carotid arteries [1,24,25,30]. Simultaneous or in-tandem developing intestinal SAM can accompany CNS SAM [24,25]. To date an accurate literature tabulation of cases is not possible since in some articles the histological diagnosis of SAM is either incorrect [31], cannot be accurately assessed [32] or is questionable [33].

The finding of venous lesions showing transmural medial muscle and intimal loss indicates that venous abdominal hemorrhages may rarely accompany SAM and should be considered in cases of persistent bleeding following catheter embolization of identified bleeding arterial lesions.

In cases of SAM arising in the absence of pre-existing entities requiring adrenergic agonist treatment, the possibility of ractopamine or clenbuterol poisoning should be considered. This can best be verified by submitting urine or plasma samples for analysis of these agents or their metabolites. Two factors should be taken in account in this analysis. First, the very short half-life of ractopamine, 4 hours in one study, with no ractopamine detected in the plasma after 24 hours [34]. This suggests that results of urine or plasma analyzed for this agent greater than one day after the onset of the disease may be meaningless if negative. Clenbuterol, however, has a much longer halflife and is more likely to be identified several days after 
the onset of SAM. Secondly, is the location of the patient. Since ractopamine has no therapeutic use in humans, exposure is most likely caused by the consumption of food animals administered this agent. However, its use as a repartitioning agent is not world-wide. It is banned in the European Union, China and its use in Taiwan and Russia is currently mired in political controversy. However, regulatory agencies in 27 countries including the United States, Japan, South Korea, Mexico, Canada, and Australia have determined that meat from animals fed ractopamine is safe for human consumption. Therefore, cases of SAM developing in the absence of pre-existing conditions requiring treatment with adrenergic agonists should be more common in countries allowing the use of ractopamine. It is my impression, based on consultation cases, that this may be true especially in Japan from where the most cases of SAM have been reported [27]. Clenbuterol, on the other hand, is illegal in the US, the European Union and China as a repartitioning agent and also for use to enhance athletic abilities. Nevertheless it is available through the internet and is principally utilized in humans as an agent to induce weight loss and build muscle. It has also been illegally used as a repartitioning agent in China and elsewhere. Clinical studies on patients poisoned by clenbuterol in food producing animals [35] have not detected SAM although some patients have developed myocardial infarcts. I suspect that some also may have developed subclinical SAM i.e., without ruptured gap-aneurysms. Follow-up angiograms of abdominal arteries would be of value in assessing whether this supposition is correct.

\section{ARTERIAL FIBROMUSCULAR DYSPLASIA CONSIDERATIONS}

Slavin and coworkers have proposed that SAM is the precursor of FMD a hypothesis derived from the morphologic appearance of SAM in its reparative phase (Figures 11 and 16-18) [7-9]. This relationship was inadvertently supported by others describing the morphology of FMD, for example, the appearance of SAM in a montage of images illustrating FMD [36]. However, this hypothesis has been contested for the following reasons. First, SAM in adults is a disease principally affecting the elderly [8], while FMD occurs in younger adults. Secondly, there is a gender discrepancy-FMD being much more frequent in females while SAM occurs with equal frequency in both sexes. Thirdly, the clinical presentations of these 2 disorders differ, SAM presenting with profuse bleeding from abdominal intestinal arteries whilst FMD is announced by ischemic changes causing hypertension or strokes derived from renal artery alterations in the former and internal carotid artery changes in the latter. Fourthly, FMD is reported in large arteries supplying the extremities a finding not definitively found in SAM. The following arguments are marshaled to counter these objections. There are 3 different arterial lesions in the injurious stage of SAM but it is the bleeding from gap-aneurysms that determines its clinical presentation. Abdominal SAM is considered a disease of the elderly because it is precisely in this group that SAM is mainly clinically recognized. The reasons why this age bracket preferentially develops this lesion may be ascribed to intense or prolonged stimuli of the alpha-1 receptors by adrenergic agonists utilized to treat concomitant circulatory disorders, a possible age-related increased sensitivity to adrenergic agents and concomitant aging changes that weaken or disrupt muscular-stromal connections thereby facilitating gap formation and enlargement [37]. The other lesions in the injurious phase, mediolysis and the tearing separation of the media from the adventitia are generally clinically silent and if present in younger patients would be asymptomatic. Gap-aneurysm formation is not a feature of infant SAM. These silent arterial injuries can remain unnoticed in the reparative phase or metamorphose to symptomatic arterial sequelae or de novo appearing fibromuscular dysplasia both causing arterial stenosis and ischemic lesions. Schouwenberg and coworkers have shown a gender differnce in cardiovascular response to infused adrenalinethe response in premenopausal women was principally alpha-adrenergic while in age matched males it was Beta-adrenergic [38]. This observation offers a possible explanation for the marked increase of FMD in adolescent and young adult females as opposed to males-the increased sensitivity of their alpha-1 adrenergic receptors to the agonists causing SAM. Suspected as causes of FMD in younger patients without medical histories is poisoning by ractopamine or clenbuterol distributed in animal muscle or viscera, diet or athletic enhancing preparations or adulterating illicit drugs such as heroin [19]. Beta-2 adrenergic tocolytic agents administered for preterm labor is another possible cause.

Is SAM the sole precursor lesion of FMD? Antecedents for perimedial, medial and adventitial FMD are clearly evident in SAM. However, since SAM does not cause intimal lesions without co-existing medial changes, the intimal form of FMD is probably not related to SAM. Moreover, reported cases of FMD principally involving arteries to or in the extremities of children, exhibit morphologic changes that do not resemble the reparative alterations of SAM [39]. FMD, therefore, is not solely caused by SAM but represents a group of arterial disorders with diverse etiologies. However, its occurrence in renal and other abdominal arteries is mainly instigated by SAM. It is not certain whether this is also true for internal carotid artery FMD, since reported cases are principally based on radiological findings. 


\section{DIAGNOSTIC CONSIDERATIONS}

Histopathological examination, although not always possible, of the affected artery is the gold standard for establishing the ultimate diagnosis of SAM, because its clinical presentation is not specific, identifying laboratory tests are absent and the radiological findings, although strongly suspicious are not absolutely definitive. Diagnostic morphologic criteria include identification of injurious stage lesions and the florid granulation tissue that repairs them. However, these criteria have pitfalls. The mostly widely utilized and misinterpreted criterion is mediolysis. Muscle cells with clear cytoplasm cannot be utilized to definitively identify mediolysis unless they exhibit features of apoptotic disintegration-nuclear pyknosis, fragmentation and loss and accompanying degenerative cytoplasmic changes featuring membranous and organelle debris dispersed in a watery cell sap and cell membrane injury with disappearance. The more classic form of apoptosis_-granular cellular disintegration also occurs in SAM but this is uncommon. Its tiny formed cellular fragments may be difficult to distinguish from the cellular debris created by the apoptotic mitochondrial injury and both may disappear rapidly or be concealed by fibrin and microhemorrhages. The latter two, and especially the linear fibrin band at the adventitial medial junction, are essential components of injurious stage SAM, and together with surviving islands of normal smooth muscle cells and occasional leukocytes create important diagnostic clues in verifying mediolysis. Indeed, in their absence the diagnosis of SAM in its injurious phase becomes problematic. Mediolysis begins in the outer media so that areas of muscle loss discovered solely in the mid or inner portion of the media sparing the outer media are not caused by SAM. Moreover, zones of medial muscle loss, replaced by expanded extracellular matrix material (see below), also is not a diagnostic features of SAM since such spaces in SAM are rapidly reoccupied by granulation tissue. Distinguishing the arterial gap from an aneurysm formed by other entities is its linear fibrin layer outline occasionally bordered by fibroblasts. Finally dissecting hematomas formed in SAM are generally not associated with intimal tears. They are recognized in the injurious phase by there origins from adjacent gaps and those in the reparative phase by their formation in bleeding granulation tissue located in the expanded separation space between the outer media and adventitia. The identification of mature granulation tissue in areas of medial muscle loss is very helpful in recognizing reparative stage SAM (Figure 13). Another morphologic hallmark of SAM is the variety of lesions-the entire panoply of injurious lesions-that may occur together in the same or adjacent arteries brought about by their asynchronous maturation, varying intensities and segmental distribution.

\section{DIFFERENTIAL DIAGNOSIS}

The principle clinical differential diagnosis of SAM is periarteritis nodosum since this entity may resemble SAM on angiograms. The distinction between these two is of importance since treatment of the former is of no value in the management of SAM and has more importantly resulted in fatalities [40]. In contrast to SAM, the lesions in this arteritis are systemic, preferably occur at branching sites, the medial muscle loss is caused by fibrinoid necrosis that generally begins in the arterial inner coat, its inflammation is significant and pleomorphic, and arterial injury frequently results in intimal scarring with luminal narrowing, dissections rarely occurring and recorded laboratory findings support an immunologic assault. There is another entity, as yet ill-defined and presumptively attributed to degenerative aging change that has most often been morphologically confused with SAM $[4,8]$. This lesion is also characterized by a noninflammatory loss of medial muscle, probably also apoptotic in type, but the vacuolar mitochondrial change described in SAM is inconspicuous (Figures 26 and 27). It is descriptively termed muscular artery cystic necrosis (MACN). MACN begins in the mid-media (Figure 28) to eventually involve most of the media although the outer media may be the last lost. In contrast to SAM no muscle cells are spared degenerative change in fields of medial loss. Expanded alcian-blue positive extracellular matrix material fills areas of muscle loss explaining the cystic component to the definition of this entity (Figures 27 and 29) This material spread thin on slides contracts to artificially form pseudovacuoles with clear contents that, when abutting muscle cells, may be mistaken for the vacuolar change in mediolysis. This change may also occur in the pools of alcian-blue material in cystic medial necrosis of the aorta. The medial change in MACN is not

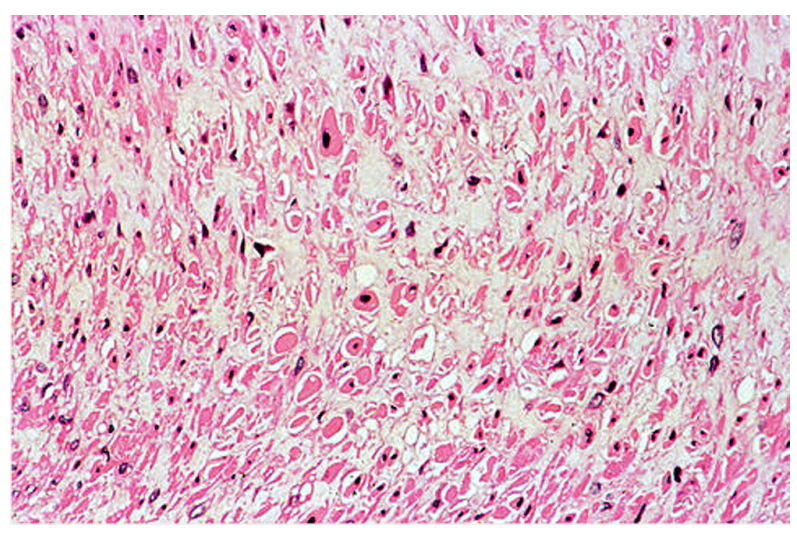

Figure 26. Muscular artery cystic necrosis. Medial muscle cells show non-inflammatory degenerative changes-nuclear pyknosis, fragmentation and loss, and hypereosinophilia, shrinkage and loss of the cytoplasm. The intervening extracellular matrix has expanded into space caused by muscle loss. Hematoxylin and eosin $\times 200$. 


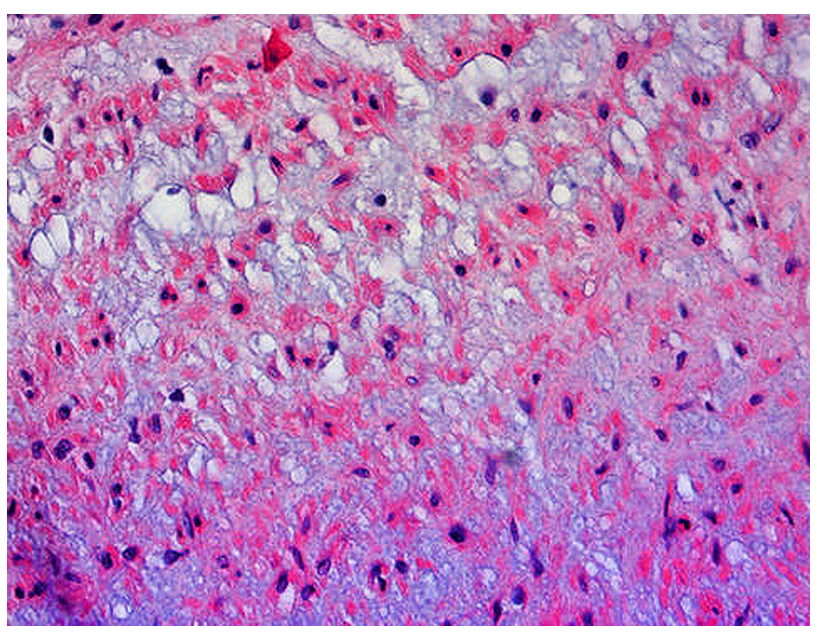

Figure 27. Muscular artery cystic necrosis. Smooth muscle cells show the degenerative changes described in Figure 26. The expanded extracellular matrix material stained with alcian-blue exhibits vacuoles with clear contents some abutting on degenerating muscle cell, a change that may be confused with the vacuolar muscle change in SAM. Alcian-blue $\times 200$.

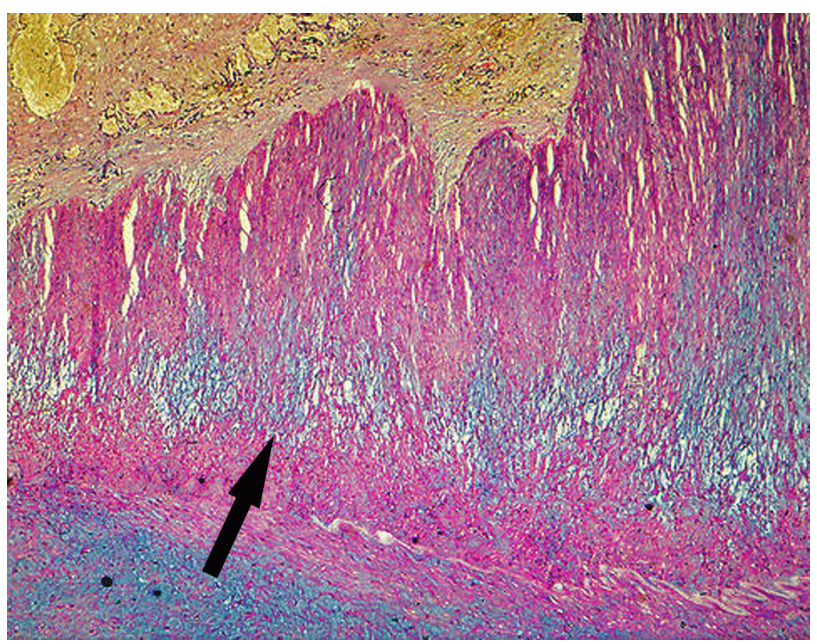

Figure 28. Muscular artery cystic necrosis. Arrow points to mid-medial cystic necrosis with focal extension toward the intima. Alcian-blue $\times 25$.

accompanied by the essential reactive components of SAM, the linear fibrin deposition at the adventitial medial junction or within the media and adventitial microhemorrhages. Separation of the adventitia from the outer media does not occur, gaps do not develop and areas of mediolysis are not reoccupied by a robust proliferation of granulation tissue. Total medial loss creates false aneurysms that may rupture and bleed (Figure 30). Dissections, reported in the literature, develop but these stem from intimal tears and some tract into the subintimal area of the affected artery [31]. MACN has been identified in the abdominal muscular, iliac, internal carotid and basilar cerebral arteries [4,31,41]. In two referral cases MACN was discovered at the transitional zone of the elastic ar-

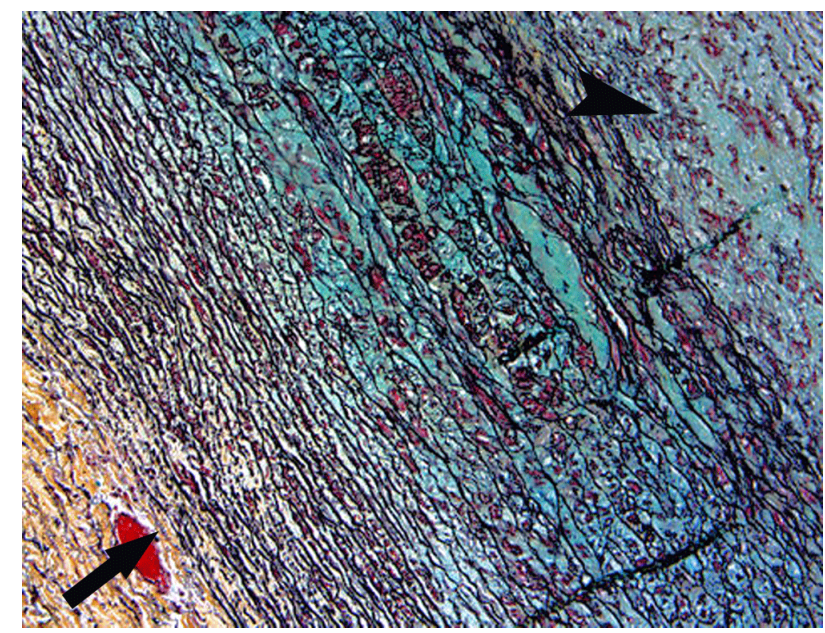

Figure 29. Muscular artery cystic necrosis. Elastic artery (arrow) shows changes of cystic medial necrosis-medial muscle loss, collapse and fragmentation of the elastic lamellae and pools of alcian-blue positive ground substance. Overlying is a muscular artery (arrow head) showing medial muscle loss and a markedly expanded alcian-blue positive ground substance in which small vacuoles with clear contents are distributed. Movat stain $\times 50$.

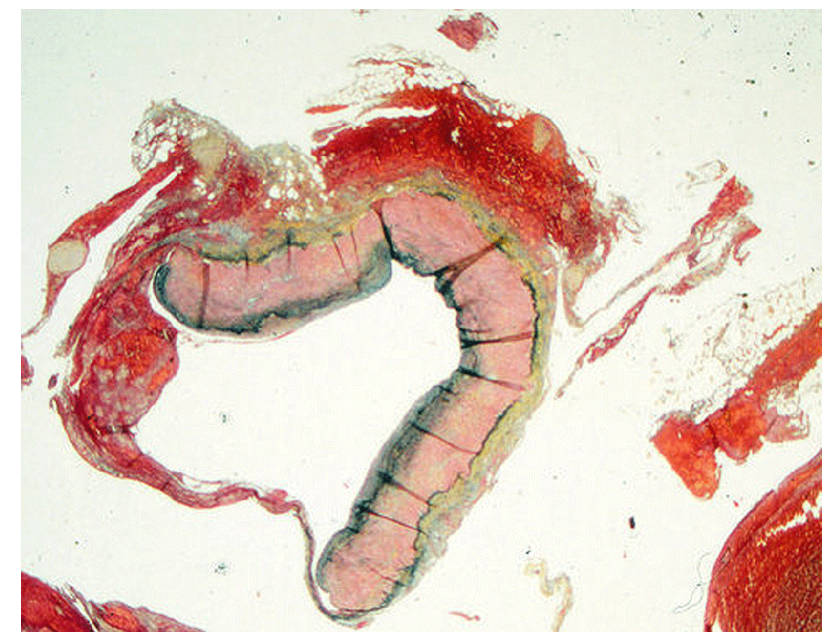

Figure 30. Muscular artery cystic necrosis. Ruptured aneurysm formed in the splenic artery showing changes of muscular artery cystic necrosis. Movat stain $\times 25$.

tery to the muscular artery, one involving the aorta and superior mesenteric artery, the other in the carotid artery (Figure 29). In both these cases the elastic artery also showed changes of cystic medial necrosis. This association has been reported in the literature with the mistaken notion that the medial changes in the muscular artery were due to SAM [41]. The morphologic similarity of MACN to cystic medial necrosis and their simultaneous occurrence strongly suggests that MACN represents the muscular artery equivalent to cystic medial necrosis of the elastic arteries an observation made by Leu who mistakenly believed MACN was SAM [31]. Cystic medial 
necrosis of the aorta is the phenotypic expression of a lesion caused by a gamut of conditions. Which one of these also causes MACA is unknown but it is not aging since MACA can occur in young adults. This disease may be clinically misdiagnosed as SAM since it can present with sudden major hemorrhages, an absence of laboratory findings of a vasculitis and a pathology that suggests similar angiographic images to SAM. This distinction may be further confounded by the fact that both entities may co-exist [4]. The realization that MACN has been mistaken for SAM vitiates the assumption that the diagnosis of SAM can rest securely on only clinical and imaging findings. Although the surgical and interventional radiological treatment of this entity is probably identical to that used for SAM, its precise identity is important since, unlike SAM, its etiology, clinical course and prognosis is unknown. Therefore, morphologic studies using the above described criteria are important and should be strictly applied to distinguish this entity from SAM. Moreover, judging from the literature the diagnosis of SAM has been utilized in some reports as a diagnosis of convenience for other as yet unidentified noninflammatory muscular artery diseases causing aneurysms [42]. Further studies are needed to identify and categorize other possible non-inflammatory mediolytic muscular artery disorders.

\section{TREATMENT}

There are several approaches to the management of SAM. Patients in shock caused by massive hemorrhages require supportive treatment to maintain hemodynamic stability and homeostasis. The realization that SAM is caused by alpha-1 adrenergic receptor agonists creates a treatment conundrum. Shock should not be treated by these agents since they have the potential to intensify SAM and generate additional lesions. Indeed, this may be the reason for the development of SAM in more than one arterial bed. Emergency surgery may be necessary to resect, ligate or bypass the causative bleeding gap aneurysms. Surgery is also indicated if vital arteries are obstructed, in cases unsuitable for interventional angiography or in problematic clinical cases where a histological diagnosis is need to determine appropriate therapy [43]. An alternative treatment for aneurysms-one that is less risky than surgery-is transcatheter embolization. This procedure can be performed immediately after the bleeding site is detected by diagnostic angiography. Glue (N-butyl cyanoacrylate) may replace coils in instances of arterial tortuosity that preclude microcatheter placement a situation that may occur in arteries with "string of beads" aneurysms. The identification of norepinephrine as the direct agent causing SAM suggests a pharmacologic strategy to thwart SAM-norepinephrine antagonists. Since SAM is announced clinically by already developed gap- aneurysms, treatment with these antagonists may only be useful in suppressing asynchronously developing lesions. Moreover, these agents may interfere with the reparative component of SAM thereby checking arterial repair and remodeling and potentially causing latent sequelae to become clinically relevant. A clinical trial is therefore necessary to evaluate the effectiveness of this pharmacological approach. Treatment with ET-1 antagonists awaits confirmation of the role played by this agent in SAM.

\section{ACKNOWLEDGMENTS}

This paper is dedicated to Professor Abou Pollack who first introduced me to SAM which he recognized as a unique arterial lesion, worthy of study, and called it arterial mediolysis, and to Dr. David Brandes who was most helpful with my first manuscript concerning SAM and named the arterial wall defects that formed in SAM gaps and finally to Dr. Micheal Yaeger, a veterinary pathologist, who by recognizing SAM in canine arteries, clinched the norepinephrine etiology of SAM. Many thanks also to Jason Slavin for his help in formatting the digital images in this chapter.

\section{REFERENCES}

[1] Kernohan, J.W. and Woltman, H.W. (1943) Postoperative, focal, nonseptic necrosis of vertebral and cerebellar arteries. JAMA, 122, 1173-1177. doi:10.1001/jama.1943.02840340021007

[2] Gruenwald, P. (1949) Necrosis in the coronary arteries of newborn infants. American Heart Journal, 38, 889-897. doi:10.1016/0002-8703(49)90889-3

[3] De Sa, D.J. (1979) Coronary arterial lesions and myocardial necrosis in stillbirths and infants. Archives of Disease in Childhood, 54, 918-930. doi:10.1136/adc.54.12.918

[4] Slavin, R.E. and Gonzales-Vitale, J.C. (1976) Segmental mediolytic arteritis: A clinical pathologic study. Laboratory Investigation, 35, 23-29.

[5] Slavin, R.E., Cafferty, L. and Cartwright, J. (1989) Segmental mediolytic arteritis: A clinicopathologic and ultrastructural study of two cases. The American Journal of Surgical Pathology, 13, 558-568. doi:10.1097/00000478-198907000-00003

[6] Lie, J.T. (1992) Segmental arterial mediolysis. Not an arteritis but a variant of arterial fibromuscular dysplasia. Archives of Pathology \& Laboratory Medicine, 116, 238241.

[7] Slavin, R.E., Sacki, K., Bhagavan, B. and Maas, A.E. (1995) Segmental arterial mediolysis: A precursor to fibromuscular dysplasia? Modern Pathology, 8, 287-294.

[8] Slavin, R.E. and Inada, K. (2007) Segmental arterial mediolysis with accompanying venous Angiopathy: A clinical pathologic review, report of 3 new cases, and comments on the role of endothelin-1 in its pathogenesis. International Journal of Surgical Pathology, 15, 121-134. doi:10.1177/1066896906297684

[9] Slavin, R.E. (2009) Segmental arterial mediolysis: Course, sequelae, prognosis, and pathologic-radiologic correlation. 
Cardiovascular Pathology, 18, 352-360. doi:10.1016/j.carpath.2008.09.001

[10] Slavin, R.E. and Yaeger, M. (2012) Segmental arterial mediolysis-An iatrogenic vascular disorder induced by ractopamine. Cardiovascular Pathology, 21, 334-338. doi:10.1016/j.carpath.2011.09.003

[11] Inayama, Y., Kitamura, H., Kitamura, H., Tobe, M. and Kanisawa, M. (1992) Segmental mediolytic arteritis clinicopathologic study and three-dimensional analysis. The Japanese Society of Pathology, 42, 201-209.

[12] Inada, K., Ikeda, K., Shimokawa, K. and Slavin, R.E. (2005) Venous lesions in segmental arterial mediolysis. Pathology and Clinical Medicine, 23, 1357-1362 (in Japanese with English abstract).

[13] Rosenberger, A., Adler, O. and Lichtig, H. (1976) Angiographic appearance of the renal vein in a case of fibromuscular dysplasia of the artery. Radiology, 118, 579580.

[14] Hashimoto, T., Degachi, J., Endo, H. and Miyata, T. (2008) Successful treatment tailored to each splanchnic arterial lesion due to segmental arterial mediolysis. Journal of Vascular Surgery, 48, 1338-1344. doi:10.1016/j.jvs.2008.05.056

[15] Anson, A. (2009) The codex perspective on ractopamine. http://www.thebeefsite.com/articles/2082/the-codex-perspective-on-ractopamine

[16] Burniston, J.G., Tan, L.B. and Goldspink, D. (2006) Relative myotoxic and haemodynamic effects of the B-agonists fenoterol and clenbuterol measured in conscious unrestrained rats. Experimental Physiology, 91, 10411049. doi:10.1113/expphysiol.2006.035014

[17] Yaeger, M.J., Mullin, K., Ensley, S., Slavin, R.E. and Ware, W.A. (2012) Myocardial toxicity in a group of greyhounds administered ractopamine. Veterinary $\mathrm{Pa}$ thology, 49, 569-573. doi:10.1177/0300985811424752

[18] Green, D.R. and Kroemer, G. (2004) The pathophysiology of mitochondrial cell death. Science, 305, 626-629. doi:10.1126/science.1099320

[19] Hoffman, R.S., Kirrane, B.M. and Marcus, S.M. (2008) A descriptive study of an outbreak of clenbuterol-containing heroin. Annals of Emergency Medicine, 52, 548-553. doi:10.1016/j.annemergmed.2008.04.026

[20] Katz, V.L. and Seeds, J.W. (1989) Fetal and neonatal cardiovascular complications from B-sympathetic therapy for tocolysis. American Journal of Obstetrics \& Gynecology, 16, 1-4.

[21] Armis, O.A. and Donovan, D.C. (1992) Segmental mediolytic arteritis involving hepatic arteries. Archives of Pathology \& Laboratory Medicine, 16, 531-534.

[22] Huntley, T.E. and Kon, V. (2001) Update on endothelinsbiology and clinical implications. Pediatric Nephrology, 16, 752-762. doi:10.1007/s004670100631

[23] Parris, R.J. and Webb, D.J. (1991) The endothelin system in cardiovascular physiology and pathophysiology. Vascular Medicine, 2, 31-43.

[24] Sakata, N., Takebayashi, S., Shimizu, K., Kojima, M., Masawa, N., Suzuki, K. and Takatama, M. (2002) A case of segmental mediolytic arteriopathy involving both in- tracranial and intraabdominal arteries. Pathology-Research and Practice, 203, 771-778.

[25] Ro, A., Kageyama, N., Takatsu, A. and Fukunaga T. (2010) Segmental arterial mediolysis of varying phases affecting both the intra-abdominal and intracranial vertebral arteries: An autopsy case report. Cardiovascular Pathology, 19, 248-251. doi:10.1016/j.carpath.2009.02.002

[26] Chao, C.P. (2009) Segmental arterial mediolysis. Seminars in Interventional Radiology, 26, 224-232. doi:10.1055/s-0029-1225666

[27] Inada, K., Maeda, M. and Ikeda, T. (2007) Segmental arterial mediolysis: Unrecognized cases culled from cases of ruptured aneurysm of abdominal visceral arteries reported in the Japanese literature. Pathology-Research and Practice, 203, 771-778. doi:10.1016/j.prp.2007.07.010

[28] Michael, M., Widmer, U., Wildermuth, S., Baghorn, A., Duewell, S. and Pfammatter, T. (2006) Segmental arterial mediolysis: CTA findings at presentation and follow-up. AJR-American Journal of Roentgenology, 187, 14631469. doi:10.2214/AJR.05.0281

[29] Lie, J.T. and Berg, K.K. (1987) Isolated fibromuscular dysplasia of the coronary arteries with spontaneous dissection and myocardial infarction. Human Pathology, 18, 654-656. doi:10.1016/S0046-8177(87)80368-4

[30] Ohtoh, T., Ono, Y., Iwasaki, Y., Sakurai, Y., Nishino, A., Arai, H., Suzuki, H. and Namba, Y. (2003) Non-traumatic recurrent dissection and its spontaneous repair in the circle of Willis: Report of two autopsy cases. Neuropathology, 23, 195-198. doi:10.1046/j.1440-1789.2003.00502.x

[31] Leu, H.J. (1994) Cerebrovascular accidents resulting from segmental mediolytic arteriopathy of the cerebral arteries in young adults. Cardiovascular Surgery, 2, 350353.

[32] Eskenasy-Cottier, A.C., Leu, H.J., Bassetti, C., Bogousslavsky, J., Regli, F. and Janzer, R.C. (1994) A case of dissection of intracranial cerebral arteries with segmental mediolytic “arteritis”. Clinical Neuropathology, 13, 329337.

[33] Fantaneanu, T., Veinot, J.P., Torres, C., Alhazzaa, M. and Stotts G. (2011) Cervical arterial dissections due to segmental mediolytic arteriopathy. Neurology, 77, 295-297. doi:10.1212/WNL.0b013e318225aad2

[34] Ungemach, F.R. (2004) Ractopamine (addendum). WHO Food Additives Series, 53.

[35] Wikipedia, the Free Encyclopedia (2012). http://enwikipedia.org/wiki/clenbuterol

[36] Vuong, P.N. and Berry, C. (2002) The pathology of vessels. Springer-Verlag, Paris.

[37] Levy, B.I. (2001) Artery changes with aging: degeneration or adoption. Dialogues in Cardiovascular Medicine, 6, 104-111.

[38] Schouwenberg, B.J., Rietjens, S.J., Smits, P. and de Galan, B.E. (2006) Effect of sex on the cardiovascular response to adrenaline in humans. Journal of Cardiovascular Pharmacology, 47, 155-157. doi:10.1097/01.fjc.0000198519.28674.cC 
[39] Price, R.A. and Vawter, G.F. (1972) Arterial fibromuscular dysplasia in infancy and childhood. Archives of $\mathrm{Pa}$ thology, 93, 419-421.

[40] Lie, J.T. (1996) Systemic cerebral and pulmonary segmental mediolytic arteriopathy; villainous masqueraders of vasculitis. Cardiovascular Pathology, 5, 305-331. doi:10.1016/S1054-8807(96)00071-3

[41] Yamada, M., Ohno, M., Itagaki, T., Takaba, T. and Matsuyama, T. (2004) Coexistence of cystic medial necrosis and segmental arterial mediolysis in a patient with aneurysms of the abdominal aorta and the iliac artery. Journal of Vascular Surgery, 39, 246-249. doi:10.1016/j.jvs.2003.07.022

[42] Basso, M.C., Flores, P.C., Marques, A., de Souza, G.L., Brescia, M.D.G., et al. (2005) Bilateral extensive cerebral infarction and mesenteric ischemia associated with segmental arterial mediolysis in two young women. Pathology International, 55, 632-638. doi:10.1111/j.1440-1827.2005.01881.x

[43] Davran, R., Cinar, C. and Parildar, M. (2010) Radiologic findings and endovascular management of three cases with segmental arterial mediolysis. CardioVascular and Intervenlional Radiology, 33, 601-606. doi:10.1007/s00270-009-9651-2 


\section{APPENDIX: (CASE PRESENTATIONS OF FIGURES)}

Case 1 [5]. The patient was an 87-year-old man, on multiple medications for cardiac arrhythmias, chronic obstructive pulmonary disease, peptic ulcer disease and mild hyperthyroidism. Approximately one month prior to admission he developed intermittently recurring vague abdominal pain that 2 days prior to admission became diffuse and severe and was accompanied by nausea, and vomiting of bilious blood flecked fluid. He was found to have a distended, tympanic and diffusely tender abdomen with guaiac-positive stools on admission to hospital. Radiographic examination of the abdomen was interpreted as representing small-bowel obstruction. At surgery a $27.5 \mathrm{~cm}$ segment of dark, erythematous, indurated jejunum was resected. On surgical pathologic examination ischemic enteritis was diagnosed. Foci of SAM were also discovered in the resected bowel. A work-up for a systemic vasculitis was negative with no evidence of a serositis, arthritis, skin or renal lesions. His postoperative course was stormy, but there was no recurrence of abdominal pain. After discharge the patient remained in his usual state of health and had no further gastrointestinal problems.

Comment: SAM in this patient was in the injurious phase with formation of transmural mediolysis and gaps

(Figures 2, 3 and 6). There were no abdominal hemorrhages and neither gap-aneurysms nor dissections were evident. SAM was not responsible for the ischemic changes since it was found in the submucosa beneath normal appearing bowel. Unknown were medications and anesthesia given to the patient on admission to hospital and prior to surgery.

Case 2 [8]. A 62-year-old male presented to hospital with symptoms of small-bowel obstruction. Six weeks before admission he had undergone a laparoscopic splenectomy for treatment of refractory thrombocytopenia. Surgical exploration revealed a 50-cm segment of congested jejunum associated with a filmy band adhesion along the accompanying mesentery. A bout of hypotension treated with dopamine preceded the segmental jejunal resection. No further abdominal complications developed after surgery.

Comment: SAM in this patient (Figure 1) was in injurious phase without gaps and very focal in the resected segment of jejunum that principally showed ischemic change. It was putatively induced by the dopamine administered to the patient hours before surgery. It was entirely asymptomatic and serendipitously found in one of many sections taken of the resected jejunum.

Case 3 [7]. A 3-year-old female child was admitted for repair of a right diaphragmatic hernia with herniation of the liver into the right thoracic cavity. Her postoperative course was complicated by aspiration, eventually treated with mechanical respiration. Further pulmonary complications followed including respiratory distress syndrome, pneumothoraces, pulmonary interstitial emphysema and pleural effusions. She was transferred to another institution and placed on extracorporeal membrane therapy for 252 hours. During this period she developed renal dysfunction and ascites and was treated with volume infusions, diuretics and a low-dose dopamine drip. She exhibited signs and symptoms of intracerebral and subarachnoid hemorrhage and subsequent cerebral blood scans documented absence of cerebral blood flow. The patient was declared brain dead, mechanical ventilation was discontinued and the patient rapidly expired.

Comment: Injurious SAM was found exclusively involving the extramural coronary arteries (Figure 4). Gaps, aneurysms, and dissections were not evident. The basilar cerebral arteries were free of SAM. The continuous low dose dopamine drips putatively caused SAM in this child.

Case 4 [4]. The patient is a 73-year-old female with a history of cardiovascular hypertensive disease treated with digoxin, thiazides and potassium supplements. She presented with a 3-week-history of constipation, abdominal pain, anorexia and weakness. Physical examination on admission revealed a patient in shock with a rigid abdomen, flank tenderness, and infrequent bowel sounds. She died within hours after admission. Medications given prior to and after admission as treatment for shock are unknown.

Comment: Figure 5 showing injurious phase SAM and Figure 10 showing early reparative phase SAM are both from this patient and illustrate the asynchronous maturation of SAM. This patient showed both phases of SAM in 8 different abdominal arteries and their immediate tributaries. Gap-aneurysms formed in several but these did not rupture. Death was caused by a clinically unrecognized rupture of an atherosclerotic thoracic aneurysm into the right thoracic cavity and mediastinum. Multiple atherosclerotic emboli aided in the development of ischemic enterocolitis and acute pancreatitis that caused her abdominal symptoms. This patient also exhibited MACN in large intestinal arteries not showing SAM.

Case 5 [4]. An 80-year-old male with a history of hypertension underwent a perineal prostatectomy for benign prostatic hyperplasia. A hypotensive event occurred during surgery that continued into the first post-operative day. This was treated for 24 hours with Metaraminal (Aramine). Three days following surgery the patient developed sudden chest pain, cardiac arrhythmia and became comatose. His hematocrit fell from 48 to 34 and he died the next day.

Comment: Figure 7 from this patient shows two gaps in a large pancreatic artery branch of the splenic artery. 
SAM also occurred in the superior pancreatico-duodenal artery and rupture of gap-aneurysms in these arteries caused massive retroperitoneal, peripancreatic, and intramesenteric hemorrhages and death. Aramine was the putative causative agent causing the development of SAM.

Case 6 [7]. A 49-year-old female presented with the sudden onset of mid-epigastric pain that awaken her at night. She had a history of an acute myocardial infarct two years previously but her coronary arteriogram was interpreted as normal. She also had a history of Raynaud's phenomenon of many years duration. Her intestinal symptoms on admission prompted an angiographic study of her intestinal arteries. This revealed two aneurysms of the ileocolic branch of the superior mesenteric artery, a saccular aneurysm of the mid splenic artery and a fusiform aneurysm of the jejunal artery. A discolored and hemorrhagic fusiform aneurysm of the ileocolic artery was resected during an exploratory laporotomy as well as the adjacent portions off right colon and loops of small bowel because vascular compromise to these areas was suspected. Postoperatively she no longer experienced abdominal pain. Her vascular lesions prompted a work-up to rule out collagen vascular disease but this was negative. The fate of her other aneurysms is unknown.

Comment: Figure 8 shows a double aneurysm with a thrombus that accounted for ischemic changes in the resected segments of bowel. SAM was present in 3 different intestinal arteries. The agent responsible for SAM in this middle-age patient with labile pressor activity (Prinzmetal angina and Raynaud's phenomena) is unknown but in the absence of adrenergic agonists poisoning by repartitioning agents is suspected.

Case 7 [7]. A 66-year-old white female had a left frontoparietal meningioma extirpated 16 days prior to her demise. Post-operatively she developed cerebral edema, shortness of breath and densities in the left costophrenic angle. The pulmonary signs and symptoms cleared and she continued therapy for cerebral edema. On the way for a scheduled CAT scan to evaluate the status of her cerebral edema the patient became unresponsive, exhibited labored breathing and a fall in blood pressure and she expired.

Comment: Figure 11 showing SAM in the reparative phase. It was limited to the left, right and large intra hepatic hepatic arteries and was asymptomatic. The agent causing SAM most likely was introduced into the patient during surgery. The patient died of massive pulmonary emboli.

Case 8 [8]. A 57-year-old white male was admitted to hospital because of the sudden onset of gross hematuria. He had a long history of alcohol abuse and Raynaud's phenomenon. Urologic examination revealed bloody urine existing from the left kidney. A left nephrectomy was performed revealing SAM and focal cortical infarcts. The patient refused an angiographic evaluation of his abdominal arteries and subsequently was lost to followup.

Comment: Figures 12, 17 and 18 show reparative phase SAM in the large intrarenal arteries. Concomitant injurious phase SAM lesions also were evident in the extrarenal hilar arteries. This combination illustrates the asynchronous evolution of SAM. The hematuria was not caused directly by SAM but was initiated by renal infarcts developing from fibrin thrombi formed in the lumens of renal arteries distal to those showing SAM. Since the patient was not on any known medications, a repartitioning agent is suspected of being the cause of SAM. 